\title{
QCD Green Functions and their Application to Hadron Physics
}

\author{
Reinhard Alkofer \\ Institut für Physik, Universität Graz, Universitätsplatz, 5, A-8010 Graz, Austria
}

(Received on 7 November, 2006)

\begin{abstract}
In a functional approach to QCD the infrared behaviour of Landau gauge Green functions is investigated. It can be proven that the ghost Dyson-Schwinger equation implies the Gribov-Zwanziger horizon condition. Its relation to the Kugo-Ojima confinement scenario is elucidated. Positivity violation for gluons is demonstrated, and the analytic structure of the gluon propagator is studied. Quark confinement is related to an infrared divergence of the quark-gluon vertex. It is shown that in the latter various components are non-vanishing due to the dynamical breaking of chiral symmetry. As a result an infrared finite running coupling in the Yang-Mills sector is derived whereas the running coupling related to the quark-gluon vertex is infrared divergent. In Coulomb gauge QCD already the one-gluon-exchange (over-)confines. This leads to a vanishing quark propagator, and thus quarks are confined. Nevertheless colour singlet quantities derived from the quark propagator are welldefined. Especially the expression for the quark condensate proves that chiral symmetry is dynamically broken. As expected the properties of mesons can be directly calculated whereas the mass of coloured diquarks diverges, and thus diquarks are confined. The latter nevertheless possess a well-defined size. In the third part the results obtained so far will be used to formulate a covariant Faddeev approach to nucleons. The resulting amplitudes describe the quark core of the nucleon. Besides the mass of this state also the electromagnetic form factors are calculated. The results for charge radii and magnetic moments as a function of the quark current mass provide some indication what the missing pion cloud may contribute to the nucleons' properties.
\end{abstract}

Keywords: Confinement; Infrared behaviour of Gluons and Quarks; Chiral Symmetry Breaking; Nucleons

\section{ON CONFINEMENT IN THE COVARIANT GAUGE: ANALYTIC PROPERTIES OF THE LANDAU GAUGE GLUON AND QUARK PROPAGATORS}

\section{A. Motivation}

Hadrons are believed to consist of quarks and gluons. However, every attempt to break a hadron into its constituents has failed so far. Thus the situation is very much different as $e . g$. in atomic physics. There studies of seperated atomic nuclei and electrons directly verify the nature of atoms being bound states. In hadronic physics, however, the evidence of physical states being bound states is indirect. Especially the plethora of hadrons finds a natural explanation in the assumption of hadrons being composite but their constituents do not exist as free particles. This phenomenon is called confinement.

The focus of these lectures is the infrared behaviour of QCD Green functions (for recent reviews see e.g. refs. [1, 2]) and what they tell us about confinement. It will be seen that from this perspective the confinement mechanisms for gluons, quarks, and colored composites show some distinctive features. Another virtue of the presented approach is the fact that QCD Green functions can be employed directly in hadron phenomenology. This opens up a road to the ambitious aim of verifying or falsifying confinement pictures experimentally.

A property of QCD which can also be infered from the hadron spectrum is the dynamical breaking of chiral symmetry. A correct description of this non-perturbative phenomenon within functional approaches requires that the chiral Ward identities between Green functions are respected, see e.g. refs. $[1,3]$. Schemes fulfilling this condition exist, and the resulting description of meson physics is impressingly successful (although many properties of mesons still have to be understood). Recently the possibility of an ab initio description of the nucleon in functional approaches to continuum quantum field theory has opened up, and my third lecture will describe the first few steps in such a direction.

The confinement problem has proven to be notoriously difficult, see e.g. ref. [4] and references therein for a brief review of (some of) the currently investigated theories of confinement. To mention two reasons out of many why the confinement problem is especially hard to solve let me first remark that the length scale of confinement is a physical scale. Based on renormalization group (RG) considerations one can then conclude that there has to exist an RG invariant confinement scale which is related to the renormalization scale $\mu$ via the $\beta$-function [5]:

$$
\Lambda_{\text {conf }}=\mu \exp \left(-\int^{g} \frac{d g^{\prime}}{\beta\left(g^{\prime}\right)}\right) \stackrel{g \rightarrow 0}{\rightarrow} \mu \exp \left(-\frac{1}{2 \beta_{0} g^{2}}\right) .
$$

This relation clearly shows that the confinement scale possesses an essential singularity in the coupling constant $g$. Therefore it is not accessible in perturbation theory, and confinement cannot be described perturbatively.

In addition, as anticipated and also verified in the course of these lectures, confinement is related to infrared singularities. Numerical lattice Monte-Carlo calculations are always restricted to finite volumes, and infrared properties can only be investigated by carefully studying the infinite volume limit. From this remark it is obvious that, to complement the lattice approach, a non-perturbative continuum approach is highly desirable if one aims at an understanding of confinement.

\section{B. Basic Concepts}

\section{Covariant Gauge Theory}

To obtain confinement the least requirement on the fundamental fields of QCD is that they do not represent particles, 
or phrased otherwise, that they do not appear as asymptotic states in the $S$-matrix.

As a matter of fact, some relations between different confinement scenarios become most transparent in a covariant formulation which includes the choice of a covariant gauge, of course. First we note that covariant quantum theories of gauge fields require indefinite metric spaces. Abandoning the positivity of the representation space already implies to give up one of the axioms of standard quantum field theory. Maintaining the much stronger principle of locality, gluon confinement then naturally relates to the violation of positivity in the gauge field sector, see e.g. ref. [1]. A comparison to QED, where the Gupta-Bleuler prescription [6] is to enforce the Lorentz condition on physical states, is instructive. There a semi-definite physical subspace can be defined as the kernel of the (onto its positive frequency part projected) field operator $\partial_{\mu} A^{\mu}$. The physical states $|\Psi\rangle$ fulfilling

$$
\partial_{\mu} A^{\mu}|\Psi\rangle=0
$$

then correspond to (equivalence classes of) states in this subspace. Covariance implies, besides transverse photons, the existence of longitudinal and timelike ("scalar") photons in QED. The latter two form metric partners in the indefinite space: They cancel against each other in every $S$-matrix element and therefore do not contribute to observables. To be more precise: The unphysical states have to be kept when inserting a complete unity (in the language of Feynman diagrams, in loops). They destructively interfere in between amplitudes (i.e. Feynman diagrams) containing these states as asymptotic states (i.e. external lines). A simple example of such Feynman diagrams is given in fig. 1.
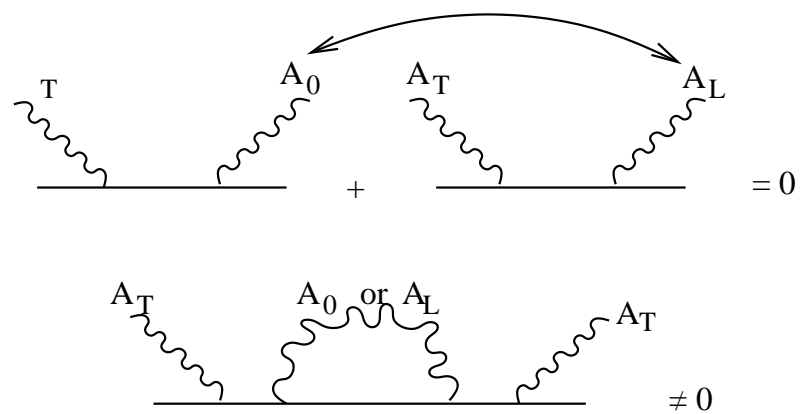

FIG. 1: An example of Feynman diagrams containing longitudinal and timelike photons as external lines (where they cancel) and in loops (where they contribute).

In QCD cancelations of unphysical degrees of freedom in the $S$-matrix also occur but are more complicated due to the self-interaction of the gluons, transverse gluons scatter into longitudinal ones and vice versa. In perturbation theory one obtains e.g. amplitudes for the scattering of two transverse into one transverse and one longitudinal gluons to order $\alpha_{S}^{2}$. A consistent quantum formulation in a functional integral approach leads to the introduction of ghost fields $c^{a}$ and $\bar{c}^{b}$ [7]. To order $\alpha_{S}^{2}$ a ghost loop then cancels all gluon loops which describe scattering of transverse to longitudinal gluons. The proof of this cancelation to all orders in perturbation theory has been possible by employing the BRST symmetry of the covariantly gauge fixed theory [8]. At this point one has achieved a consistent quantization. Also one should note that renormalizibility rests on BRST symmetry.

It is useful to picture the BRST transformation $\delta_{B}$ as a "gauge transformation" with a constant ghost field as parameter

$$
\begin{array}{r}
\delta_{B} A_{\mu}^{a}=D_{\mu}^{a b} c^{b} \lambda, \quad \delta_{B} q=-i g t^{a} c^{a} q \lambda, \\
\delta_{B} c^{a}=-\frac{g}{2} f^{a b c} c^{b} c^{c} \lambda, \quad \delta_{B} \bar{c}^{a}=\frac{1}{\xi} \partial_{\mu} A_{\mu}^{a} \lambda,
\end{array}
$$

where $D_{\mu}^{a b}$ is the covariant derivative and $\xi$ is the gauge-fixing parameter of linear covariant gauges. The parameter $\lambda$ lives in the Grassmann algebra of the ghost fields, it carries ghost number $N_{\mathrm{FP}}=-1$. Via the Noether theorem one may define a BRST charge operator $Q_{B}$ which in turn generates a ghost number graded algebra on the fields, $\delta_{B} \Phi=\left\{i Q_{B}, \Phi\right\}$. Defining the ghost number operator $Q_{c}$ one obtains

$$
Q_{B}^{2}=0, \quad\left[i Q_{c}, Q_{B}\right]=Q_{B} .
$$

This algebra is complete in the indefinite metric state space $\mathcal{V}$.

As the gauge fixing Lagrangian is BRST exact,

$$
\mathcal{L}_{G F}=\delta_{B}\left(\bar{c}\left(\partial_{\mu} A^{\mu}+\frac{\xi}{2} B\right)\right),
$$

the proof of BRST invariance of the gauge fixed action is straightforward.

The semi-definite physical subspace $\mathcal{V}_{\text {phys }}=\operatorname{Ker} Q_{B}$ is defined on the basis of this algebra by those states which are annihilated by the BRST charge $Q_{B}, Q_{B}|\psi\rangle=0$. Since $Q_{B}^{2}=0$, this subspace contains the space $\operatorname{Im} Q_{B}$ of so-called daughter states $Q_{B}|\phi\rangle$ which are images of their parent states in $\mathcal{V}$. A physical Hilbert space is then obtained as the space of equivalence classes, the BRST cohomology of states in the kernel modulo those in the image of $Q_{B}$,

$$
\mathcal{H}\left(Q_{B}, \mathcal{V}\right)=\operatorname{Ker} Q_{B} / \operatorname{Im} Q_{B} \simeq \mathcal{V}_{s} .
$$

This Hilbert space is isomorphic to the space of BRST singlets. All states are either BRST singlets or belong to quartets, this exhausts all possibilities. This generalization of the Gupta-Bleuler condition on physical states, i.e. $Q_{B}|\psi\rangle=0$, eliminates half of these metric partners from all $S$-matrix elements (leaving unpaired states of zero norm which do not contribute to any observable).

Are the transverse gluons also part of a BRST quartet? Are gluons confined this way as conjectured in refs. $[9,10]$ ? Before we return to this question it is illustrative to have a closer look into the issue of gauge fixing.

\section{Gribov horizon \& Zwanziger condition}

A detailed illustration of the issue of the non-uniqueness of gauge fixing [11] has been given in the lectures by Dan 


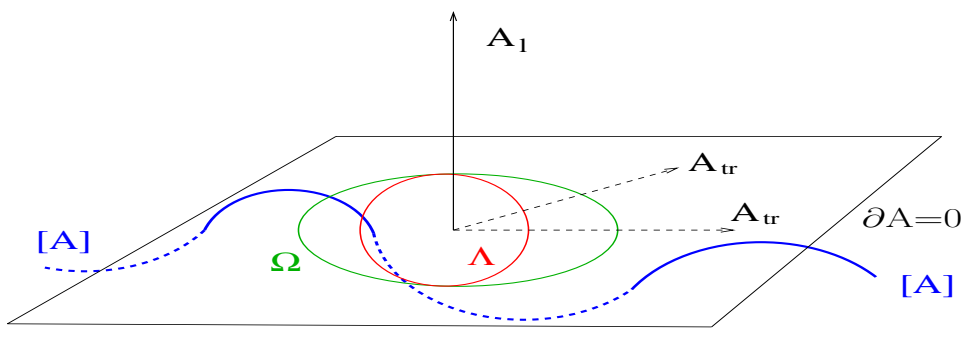

FIG. 2: A schematic representation of the configuration space of gauge fields, the "hyperplane" of transverse gauge fields, the first Gribov region and the fundamental modular region.

Zwanziger [12]. Therefore the presentation given here focuses on the implications of the infrared behaviour of QCD Green functions. Within the state of all gauge field configurations the ones fulfilling the naïve Landau gauge, i.e. the transverse gauge fields, form a "hyperplane" $\Gamma=\{A: \partial \cdot A=0\}$. As schematically illustrated in fig. 2 a gauge orbit intersects $\Gamma$ several times and therefore gauge fixing is not unique. The socalled minimal Landau gauge obtained by minimizing $\|A\|^{2}$ along the gauge orbit is usually employed in corresponding lattice calculations, it restricts the gauge fields to the Gribov region

$$
\Omega=\left\{A:\|A\|^{2} \text { minimal }\right\}=\{A: \partial \cdot A=0,-\partial \cdot D(A) \geq 0\}
$$

where the Faddeev operator $-\partial \cdot D(A)$ is strictly positive definite. Phrased otherwise: On the boundary of the Gribov region, the Gribov horizon, the Faddeev operator possesses at least one zero mode.

Unfortunately this is not the whole story. There are still Gribov copies contained in $\Omega[13,14]$, therefore one needs to restrict the gauge field configuration space even further to the region of global minima of $\|A\|^{2}$ which is called the fundamental modular region. A lot of effort has been devoted to an estimate of the corresponding effects in lattice studies, see $e . g$. [15-21]. These provided evidence that for Green functions of lattice gauge theory the effects due to Gribov copies are not too large. The situation may be even better for the continuum field theory: From an approach using stochastic quantisation Zwanziger argued that Gribov copies inside the Gribov region have no effect on the Green functions of the theory [22].

What remains to be implemented within a functional approach in continuum field theory is to cut off the functional integral over gauge fields at the boundary $\partial \Omega$ as already suggested by Gribov. The solution has been found by Zwanziger [23]: One has to require that the ghost propagator is more singular in the infrared than a simple pole,

$$
\lim _{k^{2} \rightarrow 0}\left(k^{2} D_{\text {Ghost }}\left(k^{2}\right)\right)^{-1}=0 .
$$

\section{Kugo-Ojima confinement criterion}

In the covariant gauge confinement depends on the realization of the unfixed global gauge symmetries. The identifica- tion of the BRST singlets with color singlets is possible only if the charge of global gauge transformations is BRST exact and unbroken, i.e., well-defined in the whole of the indefinite metric space $\mathcal{V}$. Then BRST singlets are the physical states and are thus constituting the physical Hilbert space $\mathcal{H}$. The sufficient conditions for this are provided by the Kugo-Ojima criterion $[9,10]$ : The current

$$
J_{\mu}^{a}=\partial_{\nu} F_{\mu \nu}^{a}+\left\{Q_{B}, D_{\mu}^{a b} \bar{c}^{b}\right\},
$$

is globally conserved, $\partial_{\mu} J_{\mu}^{a}=0$, and its two terms are of the form of a total derivative. The first term corresponds to a coboundary with respect to the space-time exterior derivative while the second term is a BRST coboundary. The corresponding charges are denoted by $G^{a}$ and $N^{a}$, respectively,

$$
Q^{a}=\int d^{3} x \partial_{i} F_{0 i}^{a}+\int d^{3} x\left\{Q_{B}, D_{0}^{a b} \bar{c}^{b}\right\}=G^{a}+N^{a} .
$$

For the first term herein there are only two possibilities, it is either ill-defined due to massless states in the spectrum of the field operator $\partial_{v} F_{\mu v}^{a}$, or else it vanishes.

In QED massless photon states contribute to the analogues of both currents in (9), and both charges on the r.h.s. in (10) are separately ill-defined. One can employ an arbitrariness in the definition of the generator of the global gauge transformations (10) to multiply the first term by a suitable constant such that these massless contributions cancel. This way one obtains a well-defined and unbroken global gauge charge which replaces the naïve definition in (10). There are two independent structures in the globally conserved gauge currents in QED which both contain massless photon contributions. These can be combined to yield one well-defined charge as the generator of global gauge transformations leaving any other combination spontaneously broken, such as the displacement symmetry which leads to the identification of the photon with massless Goldstone bosons [10].

In case the term $\partial_{v} F_{\mu \nu}^{a}$ contains no massless discrete spectrum, i.e., if there is no massless particle pole in the Fourier transform of transverse gluon correlations, one obtains $G^{a} \equiv$ 0 . In particular, this is the case for channels containing massive vector fields, i.e. in theories with Higgs mechanism. It is expected to be also the case in any color channel of QCD with confinement for which it actually represents one of the two conditions formulated by Kugo and Ojima. In both these 
situations the BRST exact charge is

$$
Q^{a}=N^{a}=\left\{Q_{B}, \int d^{3} x D_{0}^{a b} \bar{c}^{b}\right\} .
$$

The second of the two conditions for confinement is that this charge be well-defined in the whole of the indefinite metric space $\mathcal{V}$. Together these conditions are sufficient to establish that all BRST singlet physical states in $\mathcal{H}$ are also colour singlets, and that all coloured states are thus subject to the quartet mechanism. The second condition thereby provides the essential difference between Higgs mechanism and confinement. The operator $D_{\mu}^{a b} \bar{c}^{b}$ determining the charge $N^{a}$ will in general contain a massless contribution from the elementary quartet due to the asymptotic field $\bar{\gamma}^{a}(x)$ in the antighost field which is defined in the (weak) asymptotic limit: $\bar{c}^{a} \stackrel{x_{0} \rightarrow \pm \infty}{\longrightarrow} \bar{\gamma}^{a}+\cdots$. In the resulting relation

$$
D_{\mu}^{a b} \bar{c}^{b} \stackrel{x_{0} \rightarrow \pm \infty}{\longrightarrow}\left(\delta^{a b}+u^{a b}\right) \partial_{\mu} \bar{\gamma}^{b}(x)+\cdots
$$

the dynamical parameters $u^{a b}$ determine the contribution of the massless asymptotic state to the composite field operator $g f^{a b c} A_{\mu}^{c} \bar{c}^{b} \stackrel{x_{0} \rightarrow \pm \infty}{\longrightarrow} u^{a b} \partial_{\mu} \bar{\gamma}^{b}+\cdots$. These parameters can be obtained in the limit $p^{2} \rightarrow 0$ from the Euclidean correlation functions of this composite field, e.g.,

$$
\int d^{4} x e^{i p(x-y)}\left\langle D_{\mu}^{a e} c^{e}(x) g f^{b c d} A_{v}^{d}(y) \bar{c}^{c}(y)\right\rangle=:\left(\delta_{\mu v}-\frac{p_{\mu} p_{v}}{p^{2}}\right) u^{a b}\left(p^{2}\right) .
$$

The theorem by Kugo and Ojima asserts that all $Q^{a}=N^{a}$ are well-defined in the whole of $\mathcal{V}$ (and do not suffer from spontaneous breakdown), if and only if

$$
u^{a b} \equiv u^{a b}(0) \stackrel{!}{=}-\delta^{a b} .
$$

Then the massless states from the elementary quartet do not contribute to the spectrum of the current in $N^{a}$, and the equivalence between physical BRST singlet states and color singlets is established.

Within the described mechanism the physical state space of Yang-Mills theory contains only colourless states. The coloured states are not BRST singlets and therefore do not appear in $S$-matrix elements, they are unobservable. In the following I will provide evidence that the transverse gluons are BRST quartet states with gluon-ghost, gluon-antighost and gluon-ghost-antighost states in the same multiplet. Gluon confinement then occurs as kind of destructive interference between amplitudes (i.e. Feynman diagrams) containing these states as asymptotic states (i.e. external lines). The members of quartets are frequently said to be "confined kinematically". This BRST quartet mechanism can be summarized as follows:

- Perturbatively, just as in QED, one such quartet, the elementary quartet, is formed by the massless asymptotic states of longitudinal and timelike gluons together with ghosts and antighosts which are thus all unobservable.

- Non-perturbatively, and in contrast to QED, however, the quartet mechanism also applies to transverse gauge field, i.e. gluon, states. A violation of positivity (see the next subsection) for such states then entails that they are also unobservable.

In Landau gauge a sufficient criterion for relation (14) and thus for this type of confinement to occur is given by the infrared behaviour of the ghost propagator: If it is more singular than a simple pole the Kugo-Ojima confinement criterion is fulfilled [26].

Note that e.g. in maximally Abelian gauge the Kugo-Ojima scenario is not applicable [24] and thus one does not expect the ghost propagator to be IR enhanced. This has been recently confirmed in lattice calculations [25].

\section{QCD Green functions: Violation of Positivity}

Given these considerations it is obvious that if states with transverse gluons violate positivity these states do not belong to $\operatorname{Ker} Q_{B}$ and are thus not physical states. One had to conclude that the transverse gluons belong to a BRST quartet (together with a gluon-ghost, a gluon-antighost, and a 2-gluon state). Therefore it is sufficient to show positivity violation in the gluon propagator to prove this kind of gluon confinement.

At this stage it is interesting to point out that more than 25 years ago the contradiction between antiscreening of gluons (and thus asymptotic freedom) on the one hand and positivity of the gluon propagator on the other hand has been noted [27]. In QCD in linear covariant gauges one obtains the following relation for the spectral sum rule of gluon correlation function

$$
Z_{3}^{-1}=Z+\int_{m^{2}}^{\infty} d \kappa^{2} \rho\left(\kappa^{2}\right) \quad \text { with } \quad Z_{3}=\left(\frac{g^{2}}{g_{0}^{2}}\right)^{\gamma}
$$

where $Z$ is the one-particle contribution, $Z_{3}$ is the gluon renormalization constant and $\gamma$ its corresponding anomalous dimension. Due to antiscreening $Z_{3}^{-1} \rightarrow 0$, i.e. $Z_{3}^{-1} \leq Z$, and therefore one has to conclude that $\rho\left(\kappa^{2}\right) \leq 0$ for some values of $\kappa$. This means nothing else than positivity violation in the gluon propagator. However, what is needed to compellingly demonstrate such a positivity violation is to verify these arguments beyond perturbation theory.

There is another important issue which is also related to the question of positivity: How is the cluster decomposition theo- 
rem circumvented in Yang-Mills theory? Including the indefinite metric spaces of covariant gauge theories this theorem can roughly be summarized as follows [28]: For the vacuum expectation values of the local product of two field operators $A$ and $B$, being at a large spacelike separaration $R$ of each other, one obtains the following bounds depending on the existence of a finite gap $M$ in the spectrum of the mass operator [10]

$$
\begin{aligned}
& |\langle\Omega|A(x) B(0)| \Omega\rangle-\langle\Omega|A(x)| \Omega\rangle\langle\Omega|B(0)| \Omega\rangle| \leq \\
& \leq \begin{cases}\text { Const. } \times R^{-3 / 2+2 N} e^{-M R}, & \text { mass gap } M, \\
\text { Const. } \times R^{-2+2 N}, & \text { no mass gap },\end{cases}
\end{aligned}
$$

for $R^{2}=-x^{2} \rightarrow \infty$. Herein, positivity entails that $N=0$, but a positive integer $N$ is possible for the indefinite state space of gauge theories. Therefore, in order to avoid the decomposition property for products of unobservable operators $A$ and $B$ which together with the Kugo-Ojima criterion is equivalent to avoiding the decomposition property for colored clusters, there should be no mass gap in the indefinite space $\mathcal{V}$. Of course, this implies nothing on the physical spectrum of the mass operator in $\mathcal{H}$ which certainly should have a mass gap. In fact, if the cluster decomposition property holds for a product $A(x) B(0)$ forming an observable, it can be shown that both $A$ and $B$ are observables themselves. This then eliminates the possibility of scattering a physical state into color singlet states consisting of widely separated colored clusters (the "behind-the-moon" problem) [10]. It has to be noted that the Kugo-Ojima criterion implies the absence of a massless particle pole in the color charge operator, and therefore in $\partial^{v} F_{\mu v}^{a}$. This shows that the unphysical massless "excitations" which are necessary to avoid the cluster decomposition property are not the transverse gluons.

In the following compelling evidence for the above described aspects of QCD in the covariant gauge, and thus for this kind of gluon confinement, will be presented. It has to be emphasized, however, that this description is purely 'kinematical', i.e. nothing is stated about the dynamics of confinement.

\section{Infrared Exponents for Gluons and Ghosts}

The tool which will be employed to study the above raised questions is the set of Dyson-Schwinger equations (DSEs). The ones for the propagators of Landau gauge QCD are displayed in fig. 3. One sees that the propagators couple to higher $n$-point functions which are in general unknown. Thus the questions arises: What can be infered from these equations? Is it possible to derive exact results? Surprisingly the answer is: Yes, if one is willing to accept some reasonable assumptions about the mathematical properties of Green functions.

\section{An exact inequality}

It will be shown that the general properties of the ghost Dyson-Schwinger equation and one additional assumption, namely that QCD Green's functions can be expanded in

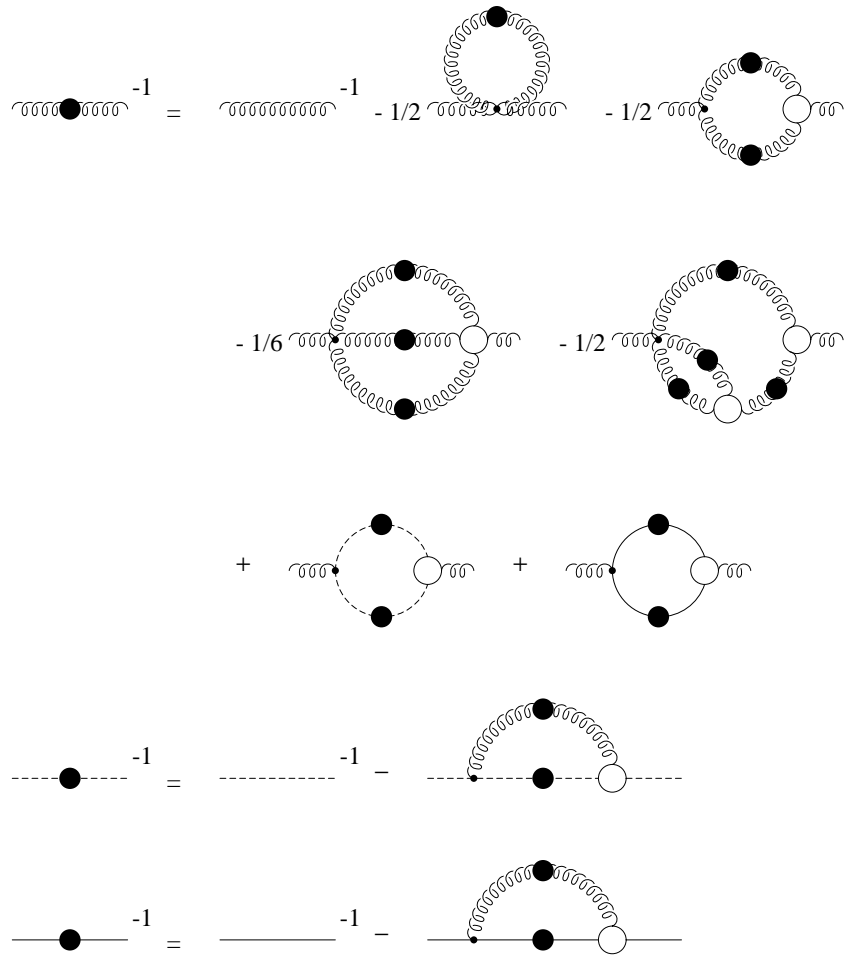

FIG. 3: Diagrammatic representation of the propagator DysonSchwinger equations. The wiggly, dashed and solid lines represent the propagation of gluons, ghosts and quarks, respectively. A filled blob represents a full propagator and a circle indicates a one-particle irreducible vertex.

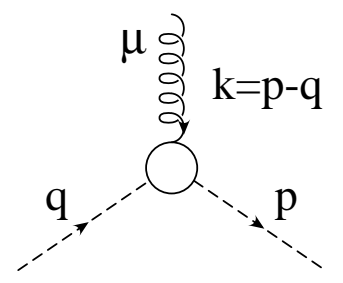

FIG. 4: Diagrammatic representation of the ghost-gluon vertex.

asymptotics series in the infrared, allow to prove the KugoOjima confinement criterion and the Gribov-Zwanziger condition [29-31].

The starting point is the non-renormalization of the ghostgluon vertex in Landau gauge to all orders in perturbation theory [32]. It does not acquire an independent ultraviolet renormalization, and even more, it stays bare for vanishing outgoing ghost momentum $p_{\mu} \rightarrow 0, c$.f. fig. 4 . These properties have been verified non-perturbatively [33-35]. Due to this the ghost-gluon vertex cannot be singular for vanishing momenta which has important consequences.

To fix the notation we note that in Landau gauge the gluon and ghost propagators are parametrized by the two invariant functions $Z\left(k^{2}\right)$ and $G\left(k^{2}\right)$, respectively. In Euclidean mo- 
mentum space one has

$$
D_{\mu v}(k)=\frac{Z\left(k^{2}\right)}{k^{2}}\left(\delta_{\mu v}-\frac{k_{\mu} k_{v}}{k^{2}}\right), \quad D_{G}(k)=-\frac{G\left(k^{2}\right)}{k^{2}} .
$$

After renormalization these propagators depend also on the renormalization scale $\mu$. Furthermore assuming that the QCD Green functions can be expanded in asymptotic series , e.g. ,

$$
G\left(p^{2} ; \mu^{2}\right)=\sum_{n} d_{n}\left(\frac{p^{2}}{\mu^{2}}\right)^{\delta_{n}}
$$

the integral in the ghost Dyson-Schwinger equation can be split in up in three pieces. The infrared integral is too complicated to be treated analytically. The ultraviolet integral, on the other hand, does not contribute to the infrared behaviour. As a matter of fact, it is the resulting equation for the ghost wave function renormalization which allows one to extract definite information [29] without using any truncation or ansatz.

The results are that the infrared behaviour of the gluon and ghost propagators is given by power laws, and that the exponents are uniquely related such that the gluon exponent is -2 times the ghost exponent. As we will see later on this implies an infrared fixed point for the corresponding running coupling. The signs of the exponents are such that the gluon propagator is infrared suppressed as compared to the one for a free particle, the ghost propagator is infrared enhanced: The infrared exponent of the ghost propagator is negative, $\kappa<0$. This exact inequality implies that the Kugo-Ojima confinement criterion and the Gribov-Zwanziger condition are both fulfilled.

It is worth emphasizing that no truncation or specialized ansatz has been used to obtain this result. The one employed assumption, namely that one is allowed to expand Green functions in asymptotic series, is not considered to be problematic. Besides this only the general structure of ghost DSE and multiplicative renormalizibility have been used. The fact that the ghost-gluon vertex is only subject to a finite renormalization turns out to be the property which makes the tower of DSEs tractable.

\section{Infrared Expansion Scheme}

Given that the Yang-Mills propagators obey infrared power laws, can one determine the infrared exponents of higher $n$ point functions? To this end the corresponding $n$-point DSEs have been studied in skeleton expansion, i.e. a loop expansion using dressed propagators and vertices, and an asymptotic expansion has ben applied to all primitively divergent Green functions [36]. It turns out that in this expansion the Green functions can only be infrared singular in the kinematical limit where all external momenta go to zero. Thus to determine the degree of possible singularities it is sufficient to investigate the DSEs in the presence of only one external $p^{2} \ll \Lambda_{\mathrm{QCD}}^{2}$. As an example consider the DSE for the threegluon vertex. In fig. 5 we see the full equation, in fig. 6 an approximation in the lowest order of a skeleton expansion. In the presence of one (small) external scale the approximated DSE has a selfconsistent power law solution given by $\left(p^{2}\right)^{-3 \kappa}$. Thus the vertex is strongly singular in the infrared. One can show by induction that this solution is also present if terms to arbitrary high order in the skeleton expansion are taken into account. Therefore the skeleton expansion provides the correct infrared solution of the DSEs.

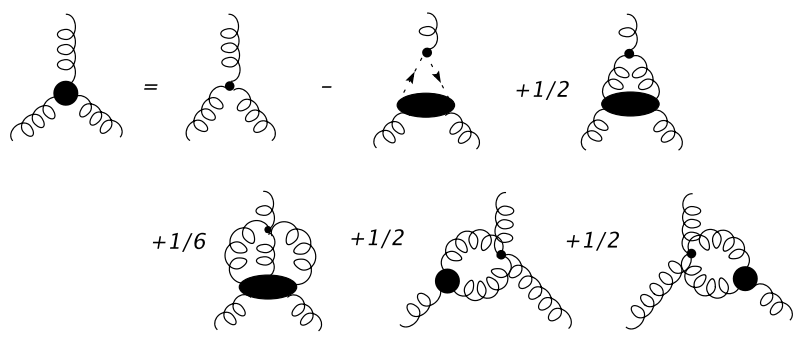

FIG. 5: Diagrammatic representation of the DSE for the 3-gluon vertex.

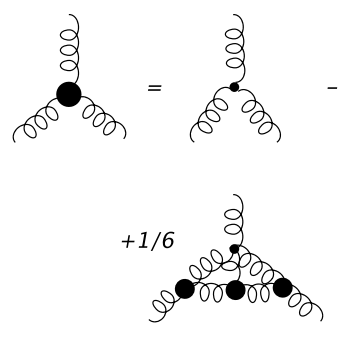

(c)

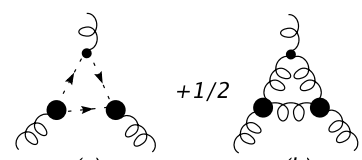

(a)

(b)

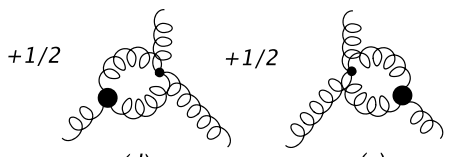

(d) (e)
FIG. 6: Diagrammatic representation of the skeleton expansion for the 3-gluon vertex.

The following general infrared (IR) behaviour for oneparticle irreducible Green functions with $2 n$ external ghost legs and $m$ external gluon legs can be derived:

$$
\Gamma^{n, m}\left(p^{2}\right) \sim\left(p^{2}\right)^{(n-m) \kappa} .
$$

Very recently it has been shown [37] by exploiting DSEs and Exact Renormalization Group Equations that this IR solution is unique. It especially includes that

- the ghost propagator is IR divergent.

- the gluon propagator is IR suppressed.

- the ghost-gluon vertex is IR finite.

- the 3- and 4-gluon vertex are IR divergent if and only if all external momenta vanish.

- every coupling from an Yang-Mills vertex possesses an IR fixed point. 


\section{Numerical results from truncated equations}

The infrared solution described above verifies the infrared dominance of the gauge fixing part of the covariantly gauge fixed QCD action conjectured in ref. [22]. The related infrared dominance of ghost loops and ultraviolet dominance of one-loop terms provides a reasoning for earlier used truncation schemes of DSEs being self-consistent at the level of two-point functions [38]. These schemes have been refined and generalized [39] and allowed then to solve the coupled set of DSEs for the ghost, gluon and quark propagators [40]. Quarks will be discussed in the next subsection, we discuss first the solution of the truncated set of DSEs depicted in fig. 7.

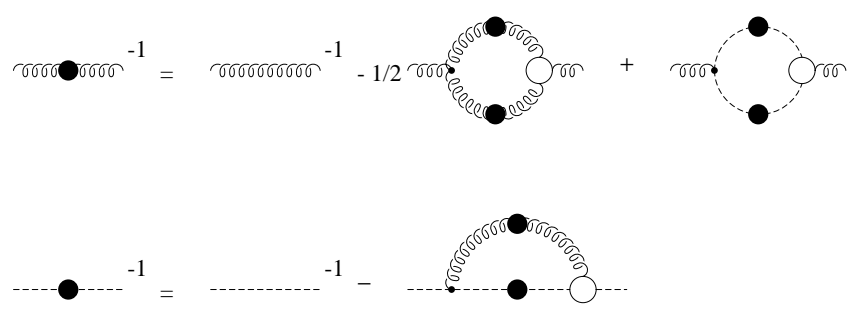

FIG. 7: Diagrammatic representation of the numerically solved truncated DSEs.

As already noted the ghost DSE (taken in this truncation fully into account) implies the IR behaviour $G\left(k^{2}\right) \rightarrow$ $g(\kappa)\left(k^{2}\right)^{-\kappa}$ and $Z\left(k^{2}\right) \rightarrow f_{1}(\kappa)\left(k^{2}\right)^{2 \kappa}$ whereas from the gluon DSE one obtains $Z\left(k^{2}\right) \rightarrow f_{2}(\kappa)\left(k^{2}\right)^{2 \kappa}$. As expected the ghost loop provides the infrared leading term. Consistency then requires

$$
f_{1}(\kappa) \stackrel{!}{=} f_{2}(\kappa) \Rightarrow \kappa=\frac{93-\sqrt{1201}}{98} \simeq 0.595353 .
$$

This result, first obtained from DSEs [30, 31], has been verified using Exact Renormalization Group Equations [41, 42].

As can be seen from refs. $[39,40]$ the numerical results for the ghost and gluon propagators compare very well to corresponding recent lattice data. However, the values of the infrared exponents extracted from lattice calculations do neither agree with the analytical obtained DSE results nor do they agree when compared against each other, see e.g. refs. $[43,44]$. A comparison to lattice calculations in three spacetime dimensions suggest that current lattice volumes are much too small for reliable extraction of infrared exponents [45].

At this point it is interesting to note that the DSEs can be solved on a compact manifold with finite volume [39, 46]. A first study of the volume dependence [46] suggested that the continuum limit would not be reached even at very large volumes. A recent investigation [47], however, has shown that ultraviolet renormalization of the DSEs is quite subtle when performed on a compact manifold. The correctly renormalized DSEs lead to numerical results which show the expected approach to the continuum limit for increasing volumes [47]. This recent development will very likely facilitate the comparison of DSE and lattice results for QCD propagators in the future.

\section{Quark Propagator and Dynamical Chiral Symmetry Breaking}

The Landau gauge quark propagator, $S(p)$, in Euclidean momentum space can be generically written as

$$
S(p)=\frac{1}{-i \not p A\left(p^{2}\right)+B\left(p^{2}\right)}=\frac{Z_{Q}\left(p^{2}\right)}{-i \not p+M\left(p^{2}\right)} .
$$

A non-vanishing mass function, $M\left(p^{2}\right)$, for vanishing current quark mass signals dynamical chiral symmetry breaking.

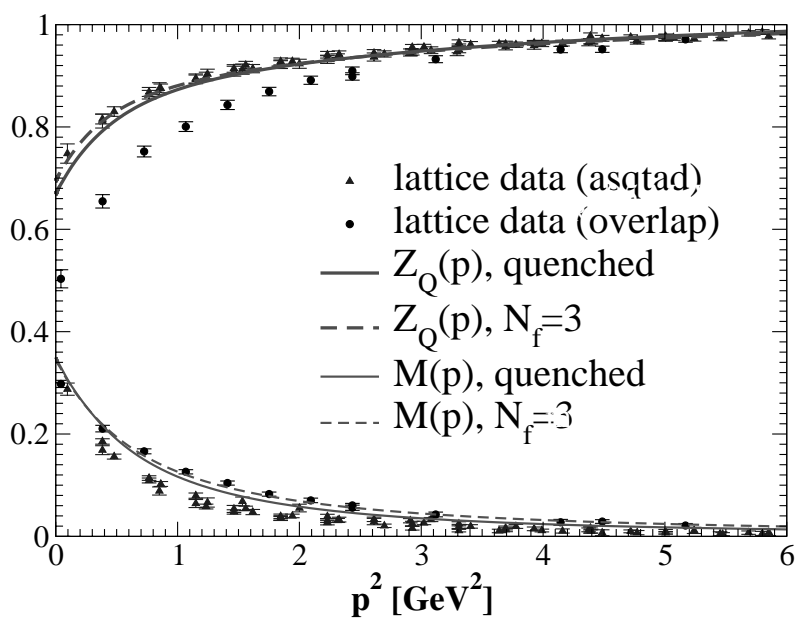

FIG. 8: The dynamically generated quark mass function $M\left(p^{2}\right)$ and the quark renormalization function $Z_{Q}\left(p^{2}\right)$ (both quenched and unquenched) as obtained from DSEs in comparison to the chiral extrapolation of quenched lattice data $[48,49]$, for details see text.

Following the arguments for the generation of a confinement scale given in the introduction one easily also verifies that the dynamical generation of quark masses is also a genuinely non-perturbative phenomenon. It furthermore requires a careful treatment of the quark-gluon interaction. In ref. [40] it is demonstrated that sizeable nontrivial Dirac structures in the quark-gluon vertex are necessary to generate dynamical quark masses of the order of 300-400 MeV. Our results for the quenched quark mass function $M\left(p^{2}\right)$ and the wave function $Z_{Q}\left(p^{2}\right)$ are compared to the chiral extrapolations of the quenched lattice results of refs. [48, 49] in fig. 8. Quite obviousy the overall qualitative and quantitative agreement between both approaches is very good. The DSE results are within the bounds given by the two different formulations of fermions on the lattice.

Including the backreaction of the quark-propagator on the ghost-gluon system leads to a coupled set of three DysonSchwinger equations for the propagators of QCD. These equations have been solved in [40] and allowed a prediction of possible effects of unquenching QCD on the propagators. Including $N_{f}=3$ chiral quarks in the gluon DSE hardly changes the results for the quark propagator, see fig. 8 .

Unquenched lattice results for the gluon propagator including the effects of two light (up-) and one heavy (strange-) 
quark have been published recently [20]. In the gluon propagator the screening effect from dynamical quarks is clearly visible in the lattice results for momenta $p$ larger than $p=0.5$ $\mathrm{GeV}$. This effect is also clearly present in the DSE results, see e.g. ref. [50].

\section{E. Running Coupling}

The definition of a non-perturbative running coupling rests on a specifically chosen Green function. As the ghost-gluon vertex in Landau gauge acquires no independent renormalisation, this relates the charge renormalisation constant $Z_{g}$ to the ones for the gluon and ghost wave functions,

$$
\widetilde{Z}_{1}=Z_{g} Z_{3}^{1 / 2} \widetilde{Z}_{3}=1,
$$

where the gluon leg provides a factor $\sqrt{Z_{3}}$, the two ghost legs $\widetilde{Z}_{3}$. As we will demonstrate in the following this allows for a definition of a non-perturbative running coupling resting solely on the properties of the gluon and ghost propagators.

From the above relations between renormalization constants one concludes that $g^{2} Z\left(k^{2}\right) G^{2}\left(k^{2}\right)$ is renormalization group (RG) invariant. In absence of any dimensionful parameter this dimensionless product is therefore a function of the running coupling $\bar{g}$,

$$
g^{2} Z\left(k^{2}\right) G^{2}\left(k^{2}\right)=f\left(\bar{g}^{2}\left(t_{k}, g\right)\right), \quad t_{k}=\frac{1}{2} \ln k^{2} / \mu^{2} .
$$

Here, the running coupling $\bar{g}(t, g)$ is the solution of $d \bar{g} / d t=$ $\beta(\bar{g})$ with $\bar{g}(0, g)=g$ and the Callan-Symanzik $\beta$-function $\beta(g)=-\beta_{0} g^{3}+O\left(g^{5}\right)$. The perturbative momentum subtraction scheme is asymptotically defined by $f(x) \rightarrow x$ for $x \rightarrow 0$. This is realized by independently setting

$$
Z\left(\mu^{2}\right)=1 \text { and } G\left(\mu^{2}\right)=1
$$

for some asymptotically large subtraction point $k^{2}=\mu^{2}$. (Note, however, that requiring both conditions is only consistent at asymptotically large $\mu^{2}$, see below.) If the quantity $g^{2} Z\left(k^{2}\right) G^{2}\left(k^{2}\right)$ is to have a physical meaning, e.g., in terms of a potential between static colour sources, it should be independent under changes $(g, \mu) \rightarrow\left(g^{\prime}, \mu^{\prime}\right)$ according to the RG for arbitrary scales $\mu^{\prime}$. Therefore,

$$
g^{2} Z\left(\mu^{\prime 2}\right) G^{2}\left(\mu^{\prime 2}\right) \stackrel{!}{=} g^{\prime 2}=\bar{g}^{2}\left(\ln \left(\mu^{\prime} / \mu\right), g\right),
$$

and, $f(x) \equiv x, \forall x$. This can thus be adopted as a physically sensible definition of a non-perturbative running coupling in the Landau gauge. In the present scheme it is not possible to realize $f(x) \equiv x$ by simply extending the perturbative subtraction scheme (24) to arbitrary values of the scale $\mu$, as this would imply a relation between the functions $Z$ and $G$ which is inconsistent with the leading infrared behaviour of the solutions. For the two propagator functions the condition (24) is in general too restrictive to be used for arbitrary subtraction points. Extending the perturbative subtraction scheme, one is only allowed to introduce functions of the coupling such that

$$
Z\left(\mu^{2}\right)=f_{A}(g) \quad \text { and } \quad G\left(\mu^{2}\right)=f_{G}(g) \quad \text { with } \quad f_{G}^{2} f_{A}=1,
$$

and the limits $f_{A, G} \rightarrow 1, g \rightarrow 0$. Using this it is straightforward to see that for $k^{2} \neq \mu^{2}$ one has $\left(t_{k}=\frac{1}{2}\left(\ln k^{2} / \mu^{2}\right)\right)$,

$$
\begin{aligned}
& Z\left(k^{2}\right)=\exp \left\{-2 \int_{g}^{\bar{g}\left(t_{k}, g\right)} d l \frac{\gamma_{A}(l)}{\beta(l)}\right\} f_{A}\left(\bar{g}\left(t_{k}, g\right)\right), \\
& G\left(k^{2}\right)=\exp \left\{-2 \int_{g}^{\bar{g}\left(t_{k}, g\right)} d l \frac{\gamma_{G}(l)}{\beta(l)}\right\} f_{G}\left(\bar{g}\left(t_{k}, g\right)\right) .
\end{aligned}
$$

Here $\gamma_{A}(g)$ and $\gamma_{G}(g)$ are the anomalous dimensions of gluons and ghosts, respectively. Eq. (22) corresponds to the following identity for these scaling functions in Landau gauge:

$$
2 \gamma_{G}(g)+\gamma_{A}(g)=-\frac{1}{g} \beta(g) .
$$

One thus verifies that the product $g^{2} Z G^{2}$ indeed gives the running coupling. Therefore the non-perturbative definition of this running coupling can be summarized as follows:

$$
\alpha_{S}\left(k^{2}\right)=\alpha_{S}\left(\mu^{2}\right) Z\left(k^{2} ; \mu^{2}\right) G^{2}\left(k^{2} ; \mu^{2}\right) .
$$

The infrared behaviour of the ghost and gluon propagators implies that the product $Z\left(k^{2}\right) G^{2}\left(k^{2}\right)$ goes to a constant in the infrared, correspondingly we find an infrared fixed point of the running coupling:

$$
\alpha_{S}(0)=\frac{2 \pi}{3 N_{c}} \frac{\Gamma(3-2 \kappa) \Gamma(3+\kappa) \Gamma(1+\kappa)}{\Gamma^{2}(2-\kappa) \Gamma(2 \kappa)} .
$$

For the gauge group $\mathrm{SU}(3)$ the corresponding numerical value is $\alpha_{S}(0) \approx 2.972$. Of course, this result depends on the employed truncation scheme. In ref. [30], assuming the infrared dominance of ghosts it has been shown that the tree-level vertex result $\alpha_{S}(0) \approx 2.972$, among the general class of dressed ghost-gluon vertices considered in the infrared, provides the maximal value for $\alpha_{S}(0)$. If the exponent $\kappa$ is chosen in an interval between 0.5 and 0.7 , as strongly suggested by lattice results, one obtains $\alpha_{S}(0)>2.5$ [30].

\section{F. Analytic properties of propagators}

\section{Positivity violation for the gluon propagator}

The positivity violation of the (space-time) propagator of transverse gluons as predicted by the Oehme-Zimmermann superconvergence relation and corresponding to the KugoOjima and Gribov-Zwanziger scenarios has been a longstanding conjecture for which there is now compelling evidence, see e.g. ref. [51] and references therein. The basic features underlying these gluon properties, are the infrared suppression of correlations of transverse gluons and the infrared enhancement of ghost correlations as discussed above. A simple argument given by Zwanziger makes this obvious: An IR vanishing gluon propagator implies for the space-time gluon propagator being the Fourier transform of the momentum space gluon propagator:

$$
0=D_{\text {gluon }}\left(k^{2}=0\right)=\int d^{4} x \quad D_{\text {gluon }}(x) .
$$




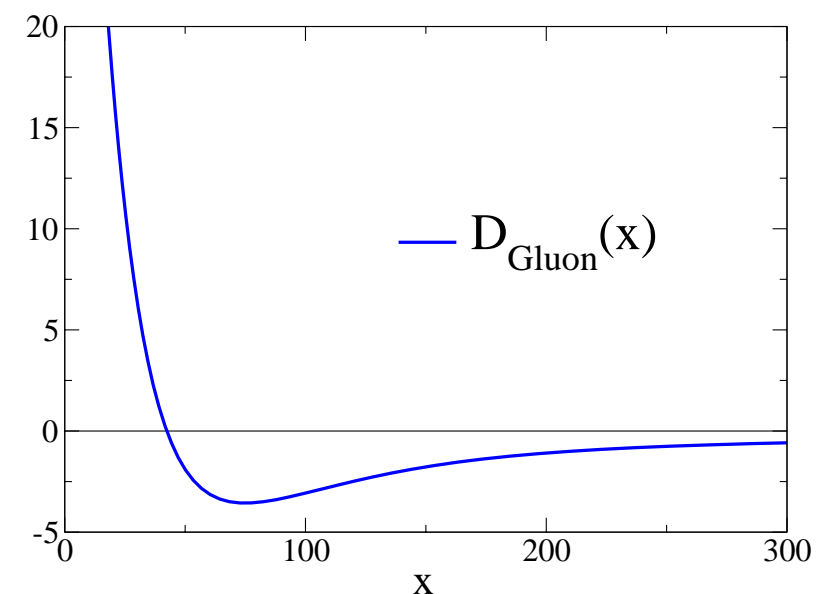

FIG. 9: The Fourier transform of the DSE result for the gluon propagator.

This implies that $D_{\text {gluon }}(x)$ has to be negative for some values of $x$. And, as a matter of fact this behaviour is seen from fig. 9 in which the Fourier transform of the DSE result for the gluon propagator is displayed.

In order to investigate the analytic structure of the gluon propagator we first parameterize the running coupling such that the numerical results for Euclidean scales are pointwise reproduced [40]:

$$
\begin{aligned}
\alpha_{\mathrm{fit}}\left(p^{2}\right) & =\frac{\alpha_{S}(0)}{1+p^{2} / \Lambda_{\mathrm{QCD}}^{2}} \\
& +\frac{4 \pi}{\beta_{0}} \frac{p^{2}}{\Lambda_{\mathrm{QCD}}^{2}+p^{2}}\left(\frac{1}{\ln \left(p^{2} / \Lambda_{\mathrm{QCD}}^{2}\right)}-\frac{1}{p^{2} / \Lambda_{\mathrm{QCD}}^{2}-1}\right)
\end{aligned}
$$

with $\beta_{0}=\left(11 N_{c}-2 N_{f}\right) / 3$. In this expression the Landau pole has been subtracted (c.f. ref. [52]), it is analytic in the complex $p^{2}$ plane except the real timelike axis where the logarithm produces a cut for real $p^{2}<0$, and it obeys Cutkosky's rule.

The infrared exponent $\kappa$ is an irrational number, and thus the gluon propagator possesses a cut on the negative real $p^{2}$ axis. It is possible to fit the solution for the gluon propagator quite accurately without introducing further singularities in the complex $p^{2}$ plane. The fit to the gluon renormalization function [51]

$$
Z_{\mathrm{fit}}\left(p^{2}\right)=w\left(\frac{p^{2}}{\Lambda_{\mathrm{QCD}}^{2}+p^{2}}\right)^{2 \kappa}\left(\alpha_{\mathrm{fit}}\left(p^{2}\right)\right)^{-\gamma}
$$

is shown in Fig. 10. Hereby $w$ is a normalization parameter, and $\gamma=\left(-13 N_{c}+4 N_{f}\right) /\left(22 N_{c}-4 N_{f}\right)$ is the one-loop value for the anomalous dimension of the gluon propagator. The discontinuity of (33) along the cut vanishes for $p^{2} \rightarrow 0^{-}$, diverges to $+\infty$ at $p^{2}=-\Lambda_{\mathrm{QCD}}^{2}$ and goes to zero for $p^{2} \rightarrow \infty$.

The function (33) contains only four parameters: the overall magnitude which due to renormalization properties is arbitrary (it is determined via the choice of the renormalization

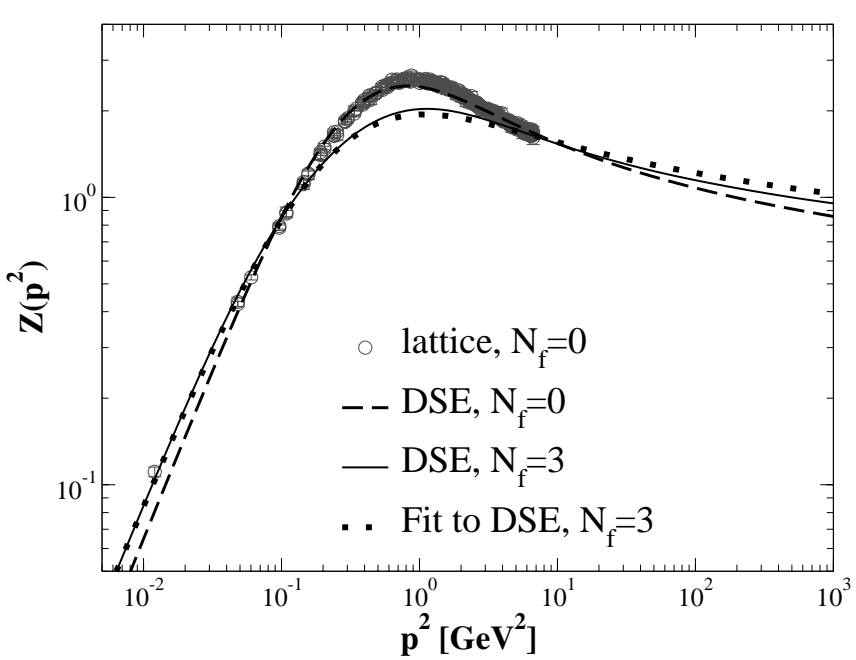

FIG. 10: The gluon propagator compared to the fit, Eq.(33), and lattice data [16].

scale), the scale $\Lambda_{\mathrm{QCD}}$, the infrared exponent $\kappa$ and the anomalous dimension of the gluon $\gamma$. The latter two are not free parameters: $\kappa$ is determined from the infrared properties of the DSEs and for $\gamma$ its one-loop value is used. Thus we have found a parameterization of the gluon propagator which has effectively only one parameter, the scale $\Lambda_{\mathrm{QCD}}$. It is important to note that the gluon propagator possesses a form such that Wick rotation is possible!

Furthermore it is worth mentioning that the positivity violations for gluons is also found at very high temperatures, even in the infinite temperature limit $[53,54]$. For the gluons being transverse to the medium the Gribov-Zwanziger and/or Kugo-Ojima scenario applies in the confined and "deconfined" phases! This should not come as a real surprise: The infinite temperature limit corresponds to three-dimensional Yang-Mills theory plus an additional Higgs-type field, the leftover of the $A_{4}$ field. The latter decouples in the IR, the threedimensional Yang-Mills theory is as expected confining and thus the corresponding gluon modes are positivity violating.

\section{Analytic structure of the quark propagator}

From the discussion above it is obvious that a dressed quark-gluon vertex is mandatory. Especially those parts of the quark-gluon vertex reflecting dynamical chiral symmetry breaking are important. Their existence provides a significant amount of self-consistent enhancement of dynamical mass generation. In ref. [51] it has been found that fairly independently of the form of the gluon propagator the resulting quark propagator respects positivity if such scalar terms are of sufficient strength in the quark-gluon vertex. The leading singularity of the quark propagator is then on the real axis, and the location of this singularity may play the role of a constituent quark mass. The results of ref. [51], however, strongly suggest that this singularity is not an isolated pole. 


\section{G. Quark Confinement}

At this point of investigations gluon confinement is inherent but quark confinement stays a mystery. Also, the precise structure of the quark propagator depends crucially on the quark-gluon vertex, and therefore a detailed study of this three-point function, and especially its IR behaviour, is required to proceed.

To extend the above described IR analysis of Yang-Mills theory to full QCD [55] one concentrates first on the quark sector of quenched QCD and chooses the masses of the valence quarks to be large, i.e. $m>\Lambda_{\mathrm{QCD}}$. The remaining scales below $\Lambda_{\mathrm{QCD}}$ are those of the external momenta of the Green functions. Without loss of generality these can be chosen to be equal, since infrared singularities in the corresponding loop integrals appear only when all external scales go to zero. One can then employ DSEs to determine the selfconsistent solutions in terms of powers of the small external momentum scale $p^{2} \ll \Lambda_{\mathrm{QCD}}$. The DSEs which have to be considered in addition to the DSEs of Yang-Mills theory are the one for the quark propagator and the quark-gluon vertex. The dressed quarkgluon vertex $\Gamma_{\mu}$ consists in general of twelve linearly independent Dirac tensors. Especially those Dirac-scalar structures are, in the chiral limit, generated non-perturbatively together with the dynamical quark mass function in a self-consistent fashion: Dynamical chiral symmetry breaking reflects itself, as anticipated by previous quark propagator DSE results, thus not only in the propagator but also in a three-point function.

An IR analysis of the full set of DSEs reveals a non-trivial solution for the quark-gluon vertex: vector and scalar components of this vertex are infrared divergent with exponent $-\kappa-\frac{1}{2}$ [55]. A numerical solution of a truncated set of DSEs confirms this infrared behavior. Similar to the Yang-Mills sector the diagrams containing ghost loops dominate. Thus all IR effects from the Yang-Mills sector are generated by the IR asymptotic theory described above. More importantly, in the quark sector the driving pieces of this solution are the scalar Dirac amplitude of the quark-gluon vertex and the scalar part of the quark propagator. Both pieces are only present when chiral symmetry is broken, either explicitely or dynamically.

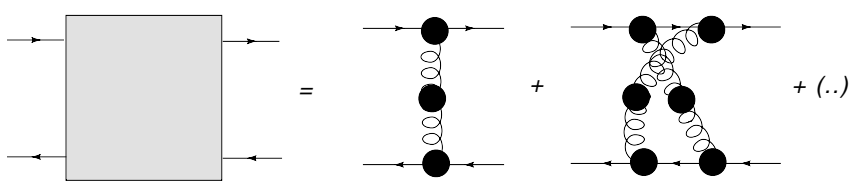

FIG. 11: The four-quark 1PI Green's function and the first terms of its skeleton expansion.

The static quark potential is obtained from the four-quark 1PI Green's function $H(p)$, whose skeleton expansion is displayed in Fig. 11. From its IR analysis one infers that $H(p) \sim$ $\left(p^{2}\right)^{-2}$ for $p^{2} \rightarrow 0$. From the well-known relation

$$
V(\mathbf{r})=\int \frac{d^{3} p}{(2 \pi)^{3}} H\left(p^{0}=0, \mathbf{p}\right) e^{i \mathbf{p r}} \sim|\mathbf{r}|
$$

between the static four-quark function $H\left(p^{0}=0, \mathbf{p}\right)$ and the quark potential $V(\mathbf{r})$ one therefore obtains a linear rising potential. Correspondingly, the running coupling from the quark-gluon vertex turns out to be proportional to $1 / p^{2}$ in the infrared, i.e. contrary to the couplings from the Yang-Mills vertices this coupling is singular in the infrared.

The first term in the skeleton expansion, i.e. the effective, nonperturbative one-gluon exchange displayed in Fig. 11, already generates this result. Since most of the terms in the expansion are equally enhanced in the IR, the string tension can only be calculated by summing over an infinite number of diagrams. This property alleviates the usefulness of the approach but it had to be expected in the first place. Since already an effective, nonperturbative one-gluon exchange generates the confining potential one is confronted with the problem of unwanted van-der-Waals forces. The suppressed gluon propagator looks at first sight helpful because it implies that there are no long-range correlations between the gauge fields, and thus no long-range correlations for chromoelctric and chromomagnetic fields at large distances. However, the problem of avoiding long-range multipole fields has only be shifted from the two-point correlation to the quark-gluon vertex.

\section{H. Summary of Part I}

The following points are the most important results described so far:

- Gluons are confined by ghosts, positivity of transverse gluons is violated.

Gluons are therefore removed from the $S$-matrix.

(c.f. the Kugo-Ojima confinement scenario, the OehmeZimmermann superconvergence relation, the GribovZwanziger horizon condition, etc.)

- Chiral symmetry is dynamically broken (in two- and three-point functions).

- In the Yang-Mills sector the strong running coupling is IR finite.

- The analytic structure of gluon and quark propagators is (very likely) such that Wick rotation is possible.

- Effectively one parameter for the gluon propagator.

- Evidence for constituent quark mass.

- There is compelling evidence that quark confinement in the Landau gauge is due to the IR divergence of the quark-gluon vertex.

In the IR this vertex is dominated by its scalar components thereby inducing a relation between confinement and broken chiral symmetry. 


\section{ASPECTS OF THE CONFINEMENT MECHANISM IN COULOMB-GAUGE QCD}

\section{A. Starting point}

As compared to the previous part of these lectures the gauge will be changed from covariant to Coulomb gauge. The aim is to relate the confinement of quarks to the confinement of coloured composites [56].

To this end we start from the commonly accepted Wilson criterion [57] and an inequality between the gauge-invariant quark-antiquark potential $V_{W}(R)$ and the color-Coulomb potential $V_{C}(\vec{x})$ [58]. The latter quantity is the instantaneous part of the time-time component of the gluon propagator in Coulomb gauge: $D_{00}(\vec{x}, t) \propto V_{C}(\vec{x}) \delta(t)+$ non-inst. terms. In ref. [58] it was shown that if $V_{W}(R)$ is confining, i.e. if $\lim _{R \rightarrow \infty} V_{W}(R) \rightarrow \infty$, then also $\left|V_{C}(\vec{x})\right|$ is confining. This was confirmed in $\mathrm{SU}(2)$ and $\mathrm{SU}(3)$ lattice calculations $[59,60]$ where it was found that $-V_{C}(\vec{x})$ rises linearly with $R=|\vec{x}|$. However, the corresponding string tension, $\sigma_{c}$, was extracted to be several times the asymptotic one, $\sqrt{\sigma_{c}} \approx 700 \mathrm{MeV}$.

Furthermore, some of the basic features of Coulomb gauge QCD will be employed. The presented investigation builds on properties of the gluon propagator in this gauge (see e.g. refs. [61-65]), investigations of the dynamical breaking of chiral symmetry in corresponding Green function approaches (see e.g. refs. [66-70]) and related results of lattice calculations (see e.g. refs. [59, 71-74]). Especially the cancellations of IR divergent expressions [69] will be exploited.

Here an instantaneous approximation is used, and the effects of transverse gluons are negelected. These approximations drastically simplify the technicalities involved in the calculations. However, some of the physics contained in the system is lost. The results are qualitatively, but not quantitatively significant. We therefore refrain from using physical dimensions, but instead present the quantities in all graphs in appropriate units of the Coulomb string tension $\sigma_{c}$. The reason for the qualitative reliability of the calculations is that the underlying symmetries of the theory are incorporated in the model via Slavnov-Taylor or Ward-Takahashi identities.

\section{B. Quark Dyson-Schwinger equation}

One immediate advantage of Coulomb gauge is the fact that it is possible to perform all calculations in Minkowski space. In the employed approximations the quark propagator fulfills the Dyson-Schwinger equation

$$
i S^{-1}(p)=\not p-m-C_{f} 6 \pi \int \frac{d^{4} q}{(2 \pi)^{4}} V_{C}(\vec{k}) \gamma_{0} S(q) \gamma_{0}
$$

where $\vec{k}=\vec{p}-\vec{q}$ and $C_{f}=\left(N_{c}^{2}-1\right) /\left(2 N_{c}\right)=4 / 3$ is the second Casimir invariant of the fundamental representation of the gauge group. The $q_{0}$-integration in Eq. (35) can be performed easily. One makes the Ansatz

$$
S^{-1}(p):=-i\left(\gamma_{0} p_{0}-\vec{\gamma} \cdot \vec{p} C(p)-B(p)\right)
$$

and obtains two coupled integral equations for the functions $B(p)$ and $C(p)$

$$
\begin{aligned}
& B(p)=m+\int \frac{d^{3} q}{2 \pi^{2}} V_{C}(k) \frac{M(q)}{\tilde{\omega}(q)} \\
& C(p)=1+\frac{1}{p^{2}} \int \frac{d^{3} q}{2 \pi^{2}} V_{C}(k) \vec{p} \cdot \vec{q} \frac{1}{\tilde{\omega}(q)}
\end{aligned}
$$

where $m$ is the current quark mass, $\tilde{\omega}(p):=\sqrt{M^{2}(p)+\vec{p}^{2}}$, and $M(p):=B(p) / C(p)$ is the quark "mass function". Its infrared behavior is a result of dynamical chiral symmetry breaking. In this Minkowski space formulation it can be directly used to define a constituent quark mass.

The Coulomb-gluon part $V_{C}(k)$ of the interaction is chosen to be highly IR singular,

$$
V_{C}(k)=\frac{\sigma_{c}}{\left(\vec{k}^{2}\right)^{2}},
$$

where $\sigma_{c}$ is the Coulomb string tension. In practical calculations $V_{C}(k)$ is regulated by a parameter $\mu_{\mathrm{IR}}$ such that the momentum dependence is modified to

$$
V_{C}(k)=\frac{\sigma_{c}}{\left(\vec{k}^{2}\right)^{2}} \rightarrow \frac{\sigma_{c}}{\left(\vec{k}^{2}+\mu_{\mathrm{IR}}^{2}\right)^{2}} .
$$

In this fashion all quantities and observables become $\mu_{\mathrm{IR}^{-}}$ dependent and one obtains the final result for some $f\left(\mu_{\mathrm{IR}}\right)$ by taking the limit $f=\lim _{\mu_{\mathrm{IR}} \rightarrow 0} f\left(\mu_{\mathrm{IR}}\right)$.

\section{Bethe-Salpeter equation}

A quark-antiquark bound state is described by the BetheSalpeter equation (BSE), which in its homogeneous form is written as (for clarity Dirac, flavor, and color indices are neglected)

$$
\Gamma(P, q)=\int d^{4} k K(q, k, P) S\left(k_{+}\right) \Gamma(P, k) S\left(k_{-}\right),
$$

where $P$ and $q$ are the quark-antiquark pair's total and relative four-momenta, $\Gamma(P, q)$ is the bound state's Bethe-Salpeter amplitude (BSA), $k_{ \pm}=k \pm P / 2$ are the individual quarkand antiquark-momenta, and $K(q, k, P)$ is the quark-antiquark scattering kernel. Note that the quark propagator appears as input into the BSE. The axial-vector Ward-Takahashi identity is employed to ensure that the kernels of the quark Dyson-Schwinger equation and Bethe-Salpeter equations for pseudoscalar states are related in such a way that chiral symmetry and its dynamical breaking are respected by the truncation. Here, corresponding to the rainbow approximation in the quark Dyson-Schwinger equation we employ the ladder approximation in the $q q$ scattering kernel in the BSE. In particular this leads to the correct behavior of the pion mass as a function of the current quark mass. Especially the pion mass vanishes in the chiral limit.

For pseudoscalar mesons and correspondingly scalar diquarks the BSA can be characterized in terms of two scalar 
functions $h(p)$ and $g(p)$, which essentially are the coefficients of the pseudoscalar and axial-vector structures in the BSA, for details, see ref. [75] and references therein. E.g. the BSE (41) leads to the following equation for the amplitude $h(p)$ :

$$
h(p)=\frac{1}{\omega(p)} \int \frac{d^{3} q}{2 \pi^{2}} V_{C}(k)\left[h(q)+\frac{m_{\pi}^{2}}{4 \omega(q)} g(q)\right],
$$

where $\omega(p)=C(p) \tilde{\omega}(p)$. Note that the same type of IR divergent integral appears as in the case of the quark DSE.

For vector mesons and axial-vector diquarks the BSA has four linearly independent amplitudes. The construction of the four coupled integral equations corresponding to the BSE is analogous to the pseudoscalar case.

\section{Cancelation of infrared divergencies}

The way the functions $B(p)$ and $C(p)$ diverge by containing a part $I(p) \propto 1 / \mu_{I R}$ makes the mass function well-defined:

$$
M(p)=\frac{I(p) M(p)+B_{r e g}(p)}{I(p)+C_{r e g}(p)}=\frac{B_{r e g}(p)}{C_{r e g}(p)} .
$$

The fact that $M(p)$ stays finite has interesting consequences: The quark propagator vanishes, i.e. quarks are confined, nevertheless the colour singlet quark condensate stays welldefined [69]. We will see that in other colour singlet quantities also the IR divergence cancels.

For a bound state two different situations may arise:

(i) The energy of a static $q \bar{q}$ state may be finite as the IR divergent quark self-energies cancel precisely against the IR divergent binding energy, or

(ii) this cancelation is incomplete, the state's energy is infinite, and it is removed from the spectrum, i.e. confined.

\section{E. Numerical results}

In fig. 12 the quark mass function $M\left(q^{2}\right)$ is plotted for four different values of the infrared regulator $\mu_{\mathrm{IR}}$ in the chiral limit $m=0$. One nicely sees the convergence of the mass function.

The BSAs for mesons also behave as expected for $\mu_{\mathrm{IR}} \rightarrow 0$. The results for the pion amplitudes $h(p)$ and $g(p)$ are presented in fig. 13 (in an arbitrary normalization such that $h(0)=1)$. As can be seen from fig. 14 the pion mass is vanishing in the chiral limit. Also the $\rho$ mass shows the expected dependence on the current quark mass.

The amplitudes for diquarks behave quite differently, the scalar diquark amplitudes $h(p)$ and $g(p)$ are presented in fig. 15. IR cancelations appearing in the pion case lead to a stable $h$ as well as ratio of $g / h$, which is not the case for the diquark: there $g / h \sim \mu_{\mathrm{IR}} \rightarrow 0$ and $h \sim 1 / \sqrt{\mu_{\mathrm{IR}}}$. This has drastic consequences for the masses when plotted vs. the IR regulating parameter $\mu_{\mathrm{IR}}$. In fig. 16 the chiral limit masses are displayed: one sees quite clearly that the $\rho$ mass is stable (the pion mass is anyhow zero) but the diquark masses are diverging for vanishing $\mu_{\mathrm{IR}}$. Therefore the diquarks are removed from the spectrum, the diquarks are confined.

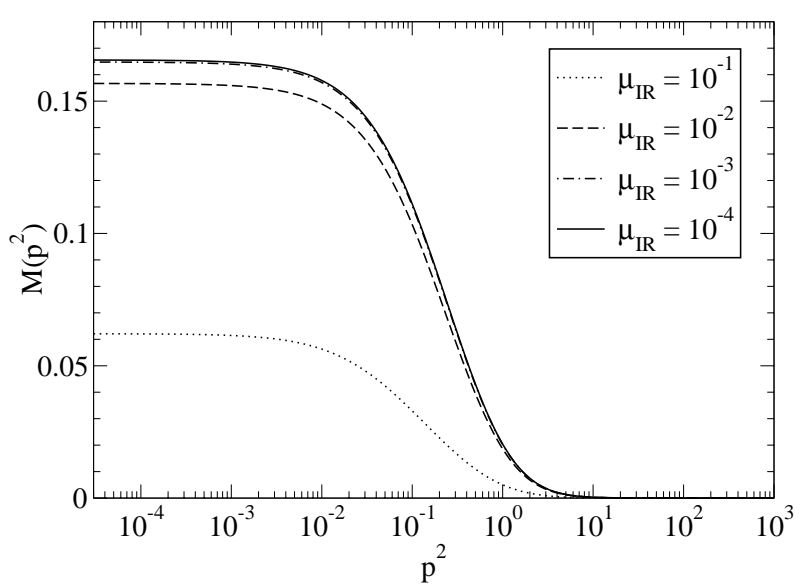

FIG. 12: The quark mass function $M\left(q^{2}\right)$ for four values of the infrared regulator $\mu_{\mathrm{IR}}$ in the chiral limit $m=0$. All quantities are given in appropriate units of $\sqrt{\sigma_{c}}$.

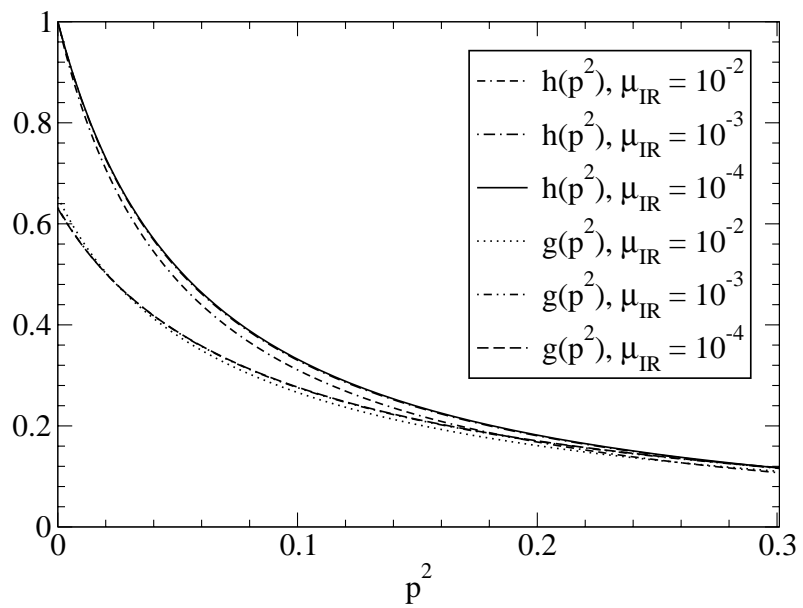

FIG. 13: Pion Bethe-Salpeter amplitude components $g$ and $h$ as functions of the infrared regulator $\mu_{\mathrm{IR}}$. For convenience, the amplitudes are normalized such that $h(0)=1$.

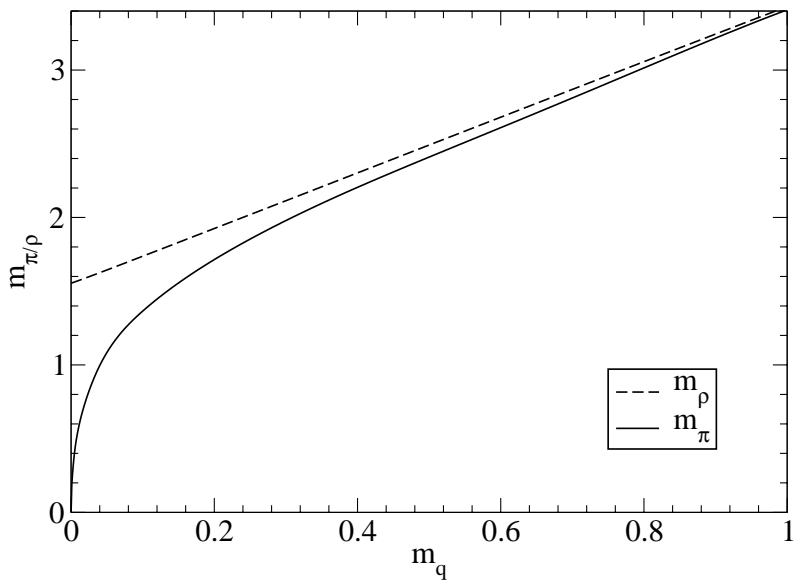

FIG. 14: The pion and rho masses as functions of the current-quark mass in the limit $\mu_{\mathrm{IR}} \rightarrow 0$. 


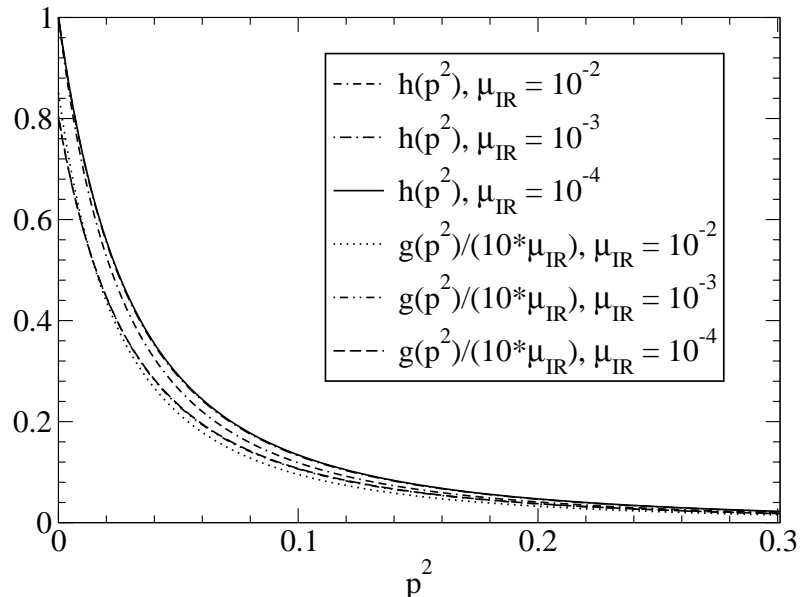

FIG. 15: Same as fig. 13 for the scalar diquark.

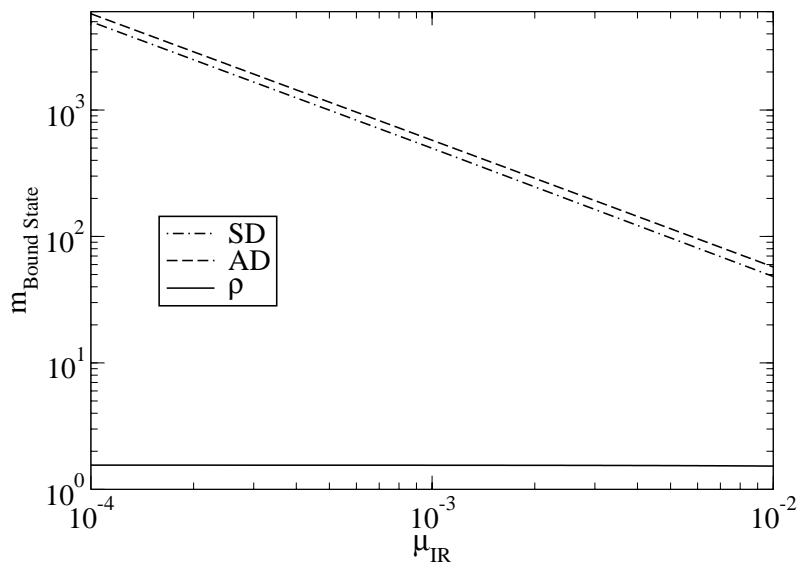

FIG. 16: The masses of the $\rho$ as well as the scalar (SD) and axialvector (AD) diquarks as functions of the infrared regulator $\mu_{\mathrm{IR}}$ in the chiral limit. The mass of the $\pi$ is identically zero for all values of $\mu_{\mathrm{IR}}$ and therefore not shown in the graph.

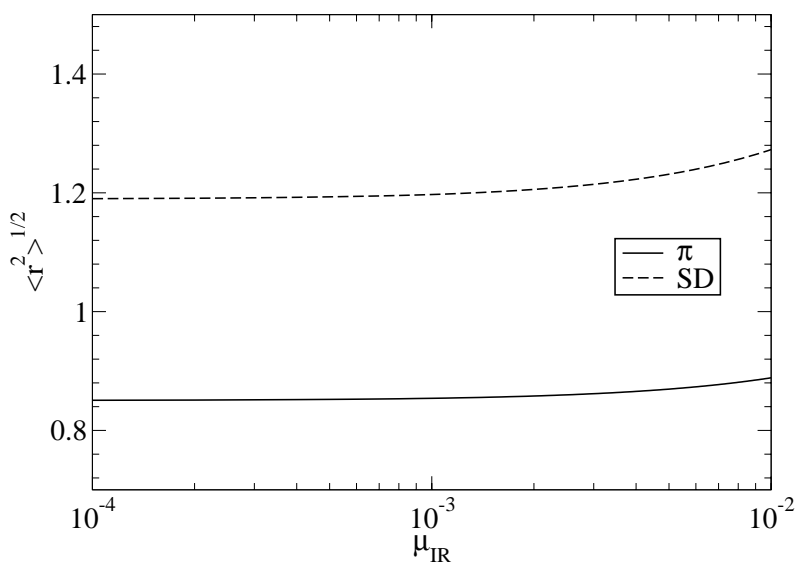

FIG. 17: Charge radii for $\pi$ meson as well as scalar diquark (SD) as functions of the infrared regulator $\mu_{\mathrm{IR}}$.
It is then quite insructive to investigate the charge radii for both meson and diquark states by calculating the electromagnetic form factors for small photon virtualities. This gives finite results in the limit $\mu_{\mathrm{IR}} \rightarrow 0$ for mesons and diquarks. Plots of the pion and scalar diquark charge radii are shown in fig. 17. We conclude therefore that the confined colour antitriplet quark-quark correlations possess a well-defined size!

\section{F. Summary of Part II}

In Coulomb gauge QCD

- the one-gluon-exchange of Coulomb-gluons ( i.e. $D_{00}$ ) overconfines!

- the (four-dimensional) quark propagator vanishes, and quarks are confined!

- dynamical chiral symmetry breaking occurs; despite IR divergencies the colour singlet quark condensate is well-defined!

- colour singlet meson properties are well-defined!

coloured diquarks are confined!

they nevertheless possess a well-defined size!

\section{WHAT IS THE NUCLEON?}

\section{A. Introduction}

The main objective of the studies to be reported here is to develop a QCD based understanding of the nucleon structure. Recent experimental results emphasize the complicated nature of baryons, and thus this aim is highly ambitious. On the other hand, as seen from the previous parts of these lectures, theoretical issues such as confinement, dynamical breaking of chiral symmetry and the formation of relativistic bound states can be understood and related to the properties of the nonperturbative propagators of QCD. This then can serve as a basis to approach an $a b$ initio calculation of nucleon properties from continuum QCD.

A first step has been recently taken [76] by progressing towards a bottom-up determination of the nucleons' quark core. Although some technical limitations could have been yet only overcome by quite drastic truncations it is worth to describe the corresponding first and quite instructive results. Within the employed Poincaré covariant Dyson-SchwingerBethe-Salpeter-Faddeev approach the building blocks are as realistic as currently available, however, yet not fully consistently determined. As will become obvious the fact that meson, especially pion, cloud effects are missing can nevertheless be clearly seen. 


\section{A note on quark models}

It has to be emphasized here that in two important respects the presented investigation differs from most quark model studies of the nucleon. First, the resulting nucleon state is a four-momentum eigenstate. This is simply due to the fact that Poincaré covariance has been respected in every step. Second, there are no parameters adjusted to any observable. Starting with DSE or lattice quark propagators this is simply not necessary. Especially, one inherits the scale generated by dimensional transmutation when non-perturbatively renormalizing QCD.

Therefore it is not intended to add to the plethora of quark models like soliton, bag or potential models. Over the last decades they have been useful in gaining a phenomenological understanding on the experimental results of the nucleon structure.

The questions to be answered by the presented approach can be considered being simple or being fundamental. Such questions are e.g.

- How do baryons interact causally?

- What rôle is played by the spin?

- Is the nucleon spherically symmetric?

Given the current situation in our understanding of the nucleon the ability to answer these questions can serve as a measure of the progress made in the investigation of hadron properties.

With these remarks in mind let us start by looking at properties of bound states of the three Dirac fermions.

\section{Relativistic angular momentum}

Even with relativistic valence quarks only, the nucleon has quite a rich structure embodied in its wave function. This will be exemplified in the nucleon's rest frame by a decomposition into partial waves w.r.t. the motion of one of the valence quarks relative to the complementary pair of quarks, see e.g. ref. [77] and references therein. As has been demonstrated this analysis also answers, without referring to a specific dynamical model, the question whether the nucleon is spherically symmetric: It is not - due to the highly relativistic motion of quarks within the nucleon.

In non-relativistic physics angular momentum is defined w.r.t. a fixed origin. Therefore the concept of angular momentum has to be generalized for relativistic states. Mathematically one sees the effect of relativity from the fact that the the Casimir operator of the non-relativistic rotation group, $\vec{J}^{2}$, does not commute with boosts.

Describing the angular momentum with the help of a vector operator orthogonal to the particle momentum will cure the underlying problem. Thus we will start our considerations from the Pauli-Lubanski axial-vector:

$$
W_{\mu}=-\frac{1}{2} \varepsilon_{\mu v \rho \sigma} J^{\mu v} P^{\sigma}
$$

where $J^{\mu \nu}$ is the Noether charge of rotations and boosts. We note that

$$
C_{2}=W_{\mu} W^{\mu}=m^{2} j(j+1)
$$

is one of the two quadratic Casimir invariants of the Poincare group, and that in the rest frame it reduces to a quantity proportional to the usual spin:

$$
W_{\mu}=(0, \vec{W}), \quad W_{i}=-\frac{1}{2} \varepsilon_{i j k 0} J^{j k} P^{0}=-m \Sigma_{i} .
$$

Note that the decomposition into spin and angular momentum is frame-dependent!

Baryons, and as such also three-quark states, are eigenstates of parity, $P^{2}$ and $W^{2}$. Coupling the three spin- $\frac{1}{2}$ quarks to a composite spin- $\frac{1}{2}$ nucleon such that Poincaré covariance is maintained we will see that [78-80]:

- due to the compositeness, we need more components than four in total or two for the positive energy states.

- the lower components will not vanish in the rest frame thus giving rise to the unavoidable presence of at least a (relativistic) $p$-wave contribution.

- the difference of one angular momentum unit between upper and lower components remains, however, there will be also a $d$-wave contribution.

- the coupling to the electromagnetic field can be chosen such to maintain causality, however, at the expense of a fairly complicated structure of the nucleon-photon vertex containing one- and two-loop contributions.

\section{Quark-quark correlations}

In a baryon every quark pair is necessarily in a colour antitriplet state. In this channel the interaction is attractive, certainly on a pertubative level and very likely also nonpertubatively. If we assume that in the corresponding rest frame the angular momentum vanishes there remain only two types of states: Scalar (Spin 0) and axialvector (Spin 1) "diquarks". Note that these are the analogous states to pseudoscalar and vector mesons because the intrinsic parity of a fermion-fermion pair is opposite to the one of a fermionantifermion pair. For these diquark states the Pauli principle requires flavour antisymmetry for the scalar and flavour symmetry for axialvector quark-quark pair.

\section{B. A Poincaré-covariant Faddeev Approach}

\section{Dyson-Schwinger equation}

The nucleon will appear as a pole in the quark six-point function. In this subsection a symbolic notation for all equations will be used, otherwise it would be hard to recognize the relevant structures under all the integrals and indices. The corresponding fully renormalized Green function obeys a DSE

$$
G=G_{0}+G_{0} K G \quad \Longleftrightarrow \quad G^{-1}=G_{0}^{-1}-K
$$


in terms of the tree-level six-point function $G_{0}$ and an interaction kernel $K, c . f$. fig. 18. This equation, in Euclidean space, provides the starting point for our investigations. In the vicinity of the pole the decomposition $G \propto \bar{\Psi} \Psi /\left(P^{2}+m_{N}^{2}\right)$ yields

$$
\Psi=G_{0} K \Psi
$$

which is diagrammatically displayed in fig. 19.

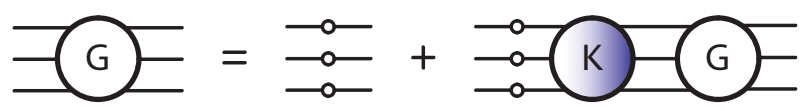

FIG. 18: A schematic representation of Dyson's equation for the quark 6-point function.

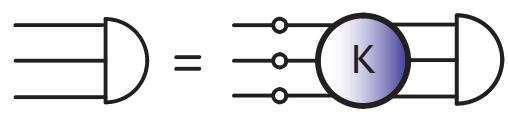

FIG. 19: The pole approximation to the DSE for the quark 6-point function.

QCD does of course also imply that irreducible threeparticle interactions exist between the three valence quarks of a nucleon. They are certainly subleading in the ultraviolet which, however, is not the reason why we neglected them here. In the previous lectures we have seen that there is a longrange confining interaction between two quarks. We then simply assume that this force is dominating the long-range correlation of also the three-quark state. When we resort now in the next step to the Faddeev approximation, namely neglecting all irreducible three-particle interactions (see fig. 20 for a diagrammatic representation) we have to be aware that this may have a drastic influence on our results. Currently this is a necessary step due to technical reasons. Only if we are able to solve the resulting Poincaré-covariant Faddeev equation, see fig. 21, we will be able to overcome this, yet uncontroled, approximation.

Provided the Faddeev approximation is justified the kernel of the 6-point DSE can be decomposed into three terms,

$$
K=K_{1}+K_{2}+K_{3} \text {. }
$$

The $K_{i}, i=1,2,3$, describe the interactions of quark pairs $(j k)$, i.e. with quark $(i)$ as a spectator. $(i j k)$ is here a cyclic permutation of (123). The two-quark propagators $g_{i}$ fulfill their own DSEs with kernels $K_{i}$,

$$
g_{i}=G_{0}+G_{0} K_{i} g_{i}
$$

The objects $g_{i}$ and $K_{i}$ are defined in three-quark space. The former contain a factor $S_{i}$, the propagator of the spectator quark, and the latter contain a factor of $S_{i}^{-1}$ (although the spectator quark is not involved in the interactions described by $K_{i}$ ). The two-quark scattering kernel $\widetilde{T}_{i}$ is defined by amputating all incoming and outgoing quark legs from the connected part of $g_{i}$,

$$
g_{i}=G_{0}+G_{0} \widetilde{T}_{i} G_{0}
$$

Combining the two previous equations yields an integral equation for $\widetilde{T}_{i}$,

$$
\widetilde{T}_{i}=K_{i}+K_{i} G_{0} \widetilde{T}_{i}
$$

The so-called Faddeev components $\psi_{i}$ are introduced by

$$
\Psi_{i}=G_{0} K_{i} \Psi
$$

which finally allows us to write down the Faddeev equation

$$
\Psi_{i}=S_{j} S_{k} \widetilde{T}_{i}\left(\Psi_{j}+\Psi_{k}\right) .
$$

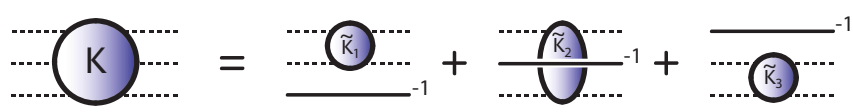

FIG. 20: Diagrammatic representation of the Faddeev approximation.

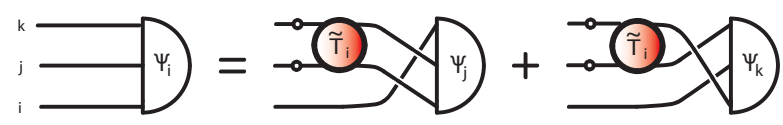

FIG. 21: The Poincaré-covariant Faddeev equation of the nucleon.

\section{Quark propagator}

In eq. (54) the fully renormalized quark propagator appears explicitely as well as implicitely (via the two-quark scattering kernel). In part I of these notes it is described how to obtain this quark propagator by solving the Landau gauge DSEs for the quark, gluon and ghost propagators as well as the quarkgluon vertex. In the calculations discussed below the parameterization of ref. [51] with a cut on the real time-like axis has been used. The quark DSE for complex external momenta, as needed in the Faddeev equation, is solved within an on-going investigation [81]. However, these results have yet not been included into a solution of the Faddeev equation. There is another advantage of using the parameterization of the DSE solution: With some very mild adjustment of parameters it also describes to a high accuracy the available lattice data for the quark propagators.

As has been discussed the dynamical chiral symmetry breaking, and thus the dynamical generation of the infrared quark mass, is quantitatively well described. The 'constituent quark' mass scale is described by $M(0) \approx 350 \ldots 400 \mathrm{MeV}$. The analytic structure is, on the other hand, quite sensitive to the details on the spacelike axis. In the chiral limit the location of the singularity closest to the origin is $M\left(p_{\text {sing }}^{2}\right) \approx 500 \mathrm{MeV}$ when using the DSE solution and $390 \mathrm{MeV}$ when adjusting 
to lattice data. This then implies finally a 'range' of quark propagator parameterizations to be used.

As the parameterization of ref. [51] is based on DSE solutions and/or lattice data with different values of the current mass the dependence of the quark propagator functions on the current masses is kept quite precisely. For some values of the current mass the constituent quark mass function is plotted in fig. 22.

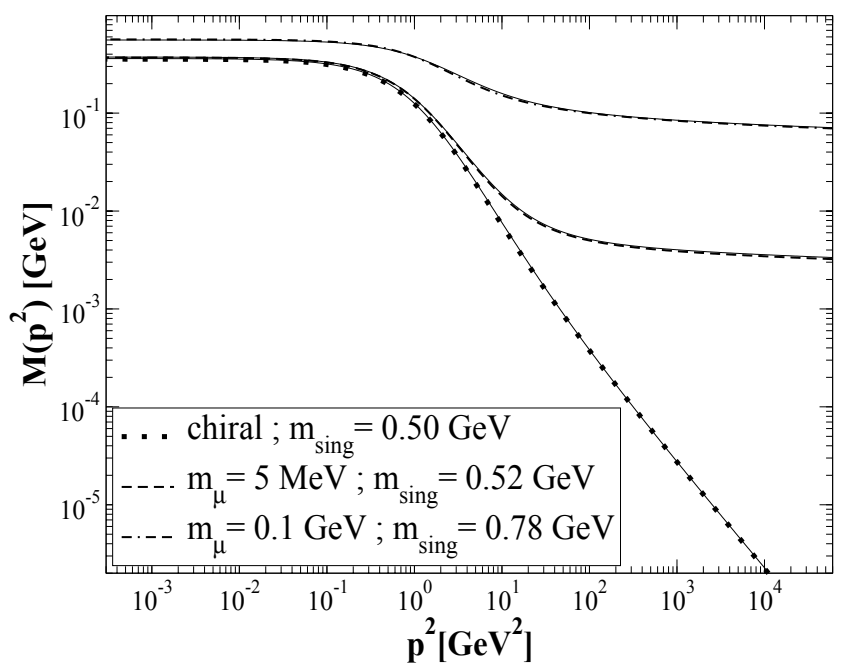

FIG. 22: The mass functions of the employed quark propagators for different values of the current mass calculated from the parameterization of ref. [51].

\section{3. "Diquarks"}

In an on-going investigation [81] the two-quark scattering kernel $\widetilde{T}_{i}$ is calculated for the complex momenta needed in the Faddeev equation. The results presented here are, however, calculated using a further approximation, namely by representing the two-quark correlation function in terms of a sum over separable correlations,

$$
\widetilde{T}_{i}=\sum_{a} \chi_{i}^{a} D_{i}^{a} \bar{\chi}_{i}^{a}
$$

which is pictorially shown in fig. 23 . Hereby $D^{a}$ denotes the

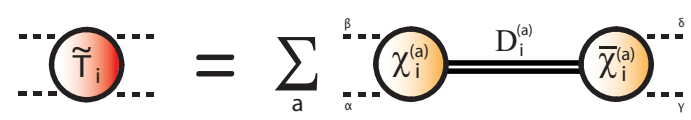

FIG. 23: Diagrammatic representation of the separable approximation to the two-quark scattering kernel $\widetilde{T}_{i}$. diquark propagator and $\chi^{a}, \bar{\chi}^{a}$ the diquark amplitudes. The sum extends over scalar and axial-vector correlations. For the amplitudes we take into account the corresponding leading Dirac structures

$$
\begin{aligned}
& \chi^{5}\left(p^{2}\right)=V_{\mathrm{sc}}\left(p^{2}\right) \gamma^{5} C, \\
& \chi^{\mu}\left(p^{2}\right)=V_{\mathrm{ax}}\left(p^{2}\right) \gamma^{\mu} C,
\end{aligned}
$$

with $C$ being the charge conjugation Dirac matrix. The amplitudes $V\left(p^{2}\right)$, reflecting the non-pointlike structure of quarkquark correlations, are determined from an 'on-shell' BetheSalpeter equation for the quark pair. In this way the confined nature of "diquarks" is not taken into account, and one obtains masses for the "diquarks". In the chiral limit, depending on the quark propagator parameterization, one obtains $m_{s c} \approx 0.6-0.8 \mathrm{GeV}, m_{a x} \approx 0.85-1 \mathrm{GeV}$. The quark pole mass and the diquark masses as a function of the current mass are plotted in fig. 24.

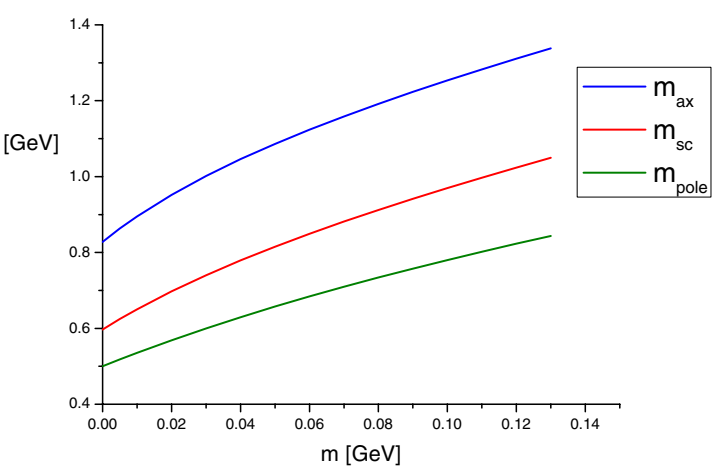

FIG. 24: Displayed are the quark pole mass and the diquark masses as a function of the current mass for parameterization of the quark propagator as resulting from coupled DSEs.

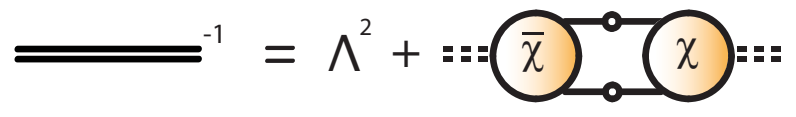

FIG. 25: Reconstruction of the diquark propagator from a quark loop.

Given the knowledge about the diquark BSAs and the diquark masses the diquark propagator can be quite accuratelly represented by the sum of a constant and a quark loop, see fig. 25. The constant is hereby chosen such that the correct "diquark msss" is reproduced.

\section{Three-quark amplitudes}

Plugging the separable approximation for the two-quark scattering kernel $\widetilde{T}_{i}$ into the Faddeev equation leads to a coupled set of quark-diquark Bethe-Salpeter equations which is 
best formulated for the the vertex functions $\Phi^{a b}$ related to the BSAs via

$$
\Psi^{a c}(p, P)=S\left(p_{q}\right) D^{a b}\left(p_{d}\right) \Phi^{b c}(p, P) .
$$

The quark-diquark Bethe-Salpeter equations then read:

$$
\phi^{a}(p, P)=\sum_{b, c} \int \frac{d^{4} k}{(2 \pi)^{4}} \underbrace{\chi^{b} S^{T}(q) \bar{\chi}^{a}}_{K_{B S}(p, k, P)} S\left(k_{q}\right) D^{b c}\left(k_{d}\right) \phi^{c}(k, P) .
$$

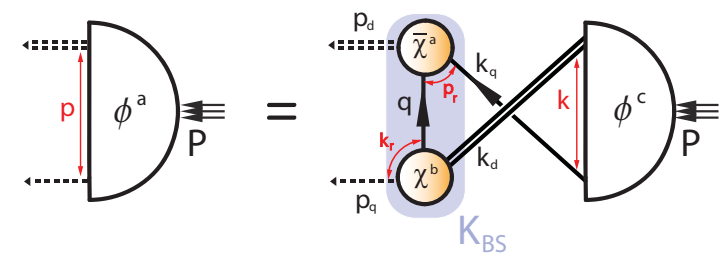

FIG. 26: Diagrammatic representation of the quark-diquark BetheSalpeter equations.

The interaction between the spectator quark and the correlated quark-quark pair is quark exchange, which is also the least interaction required to reinstate the Pauli principle. This interaction is attractive as antisymmetrisation in colour requires the symmetrisation in all other quantum numbers.

The Bethe-Salpeter vertex function for the nucleon can be decomposed into

$$
\begin{gathered}
\Phi_{\alpha \beta \gamma}^{N}=\Phi_{\alpha}^{5}\left(\chi^{5}\right)_{\beta \gamma}+\Phi_{\alpha}^{\mu}\left(\chi^{\mu}\right)_{\beta \gamma} \\
\Phi^{5}=\sum_{i=1}^{2} S_{i}(p ; P) \Gamma_{i}\left(\gamma^{\mu}, p, P\right) u \\
\Phi^{\mu}=\sum_{i=1}^{6} A_{i}(p ; P) \Gamma_{i}^{\mu}\left(\gamma^{\mu}, p, P\right) u
\end{gathered}
$$

with constraints on the Dirac matrices $\Gamma_{i}$ such that

the nucleon has spin $\frac{1}{2}$, positive parity and positive energy.

the two independent momenta

$$
\begin{aligned}
& P=\text { baryon momentum }, \text { and } \\
& p=\text { quark-diquark relative momentum, }
\end{aligned}
$$

are basis vectors.

The partial wave decomposition of the nucleon in the rest frame is given in table 1 . Besides three $s$-waves (two of them

\begin{tabular}{|c|c|c|}
\hline $\mathrm{N}$ wave fcts in the rest frame & $\begin{array}{c}\text { eigenvalue } \\
l(l+1) \text { of } \mathbf{L}^{2}\end{array}$ & $\begin{array}{c}\text { eigenvalue } \\
s(s+1) \text { of } \mathbf{S}^{2}\end{array}$ \\
\hline $\begin{array}{l}S_{1} u\left(\gamma_{5} C\right)=\left(\begin{array}{l}\chi \\
0\end{array}\right)\left(\gamma_{5} C\right) \\
S_{2} u\left(\gamma_{5} C\right)=\left(\begin{array}{l}0 \\
\text { scalar }(\vec{\sigma} \vec{p}) \chi\end{array}\right)\left(\gamma_{5} C\right)\end{array}$ & $\begin{array}{l}0 \mathrm{~s} \\
2 \mathrm{p}\end{array}$ & $\begin{array}{l}\frac{3}{4} \\
\frac{3}{4}\end{array}$ \\
\hline $\mathcal{A}_{1}^{\mu} u\left(\gamma^{\mu} C\right)=\hat{P}^{0}\left(\begin{array}{c}\frac{1}{p}(\vec{\sigma} \vec{p}) \chi \\
0\end{array}\right)\left(\gamma^{4} C\right)$ & $2 \mathrm{p}$ & $\frac{3}{4}$ \\
\hline $\mathcal{A}_{2}^{\mu} u\left(\gamma^{\mu} C\right)=\hat{P}^{0}\left(\begin{array}{l}0 \\
\chi\end{array}\right)\left(\gamma^{4} C\right)$ & $0 \mathrm{~s}$ & $\frac{3}{4}$ \\
\hline $\begin{aligned} \mathcal{B}_{1}^{\mu} u\left(\gamma^{\mu} C\right)=\left(\begin{array}{c}i \sigma^{i} \chi \\
0\end{array}\right)\left(\gamma^{i} C\right) & \text { axialvector }\end{aligned}$ & $0 \mathrm{~s}$ & $\frac{3}{4}$ \\
\hline $\mathcal{B}_{2}^{\mu} u\left(\gamma^{\mu} C\right)=\left(\underset{\frac{i}{p} \sigma^{i}(\vec{\sigma} \vec{p}) \chi}{0}\right)\left(\gamma^{i} C\right)$ & $2 p$ & $\frac{3}{4}$ \\
\hline$C_{1}^{\mu} u\left(\gamma^{\mu} C\right)=\left(\begin{array}{c}i\left(\hat{p}^{i}(\hat{\sigma} \hat{p})-\frac{1}{3} \sigma^{i}\right) \chi \\
0\end{array}\right)\left(\gamma^{i} C\right)$ & $6 \mathrm{~d}$ & $\frac{15}{4}$ \\
\hline$C_{2}^{\mu} u\left(\gamma^{\mu} C\right)=\left(\underset{\frac{i}{p}\left(p^{i}-\frac{1}{3} \sigma^{i}(\vec{\sigma} \vec{p})\right) \chi}{0}\right)\left(\gamma^{i} C\right)$ & $2 p$ & $\frac{15}{4}$ \\
\hline
\end{tabular}
also present in the non-relativistic limit) some of the lower components provide $p$-waves (four in total), and there is also a $d$-wave component. The existence of the latter means that the nucleon is, as a quantum state of course only in the internal frame, not spherically symmetric. The angular momentum coupling is depicted in fig. 27.
TABLE I: The partial wave decomposition of the nucleon in its rest frame.

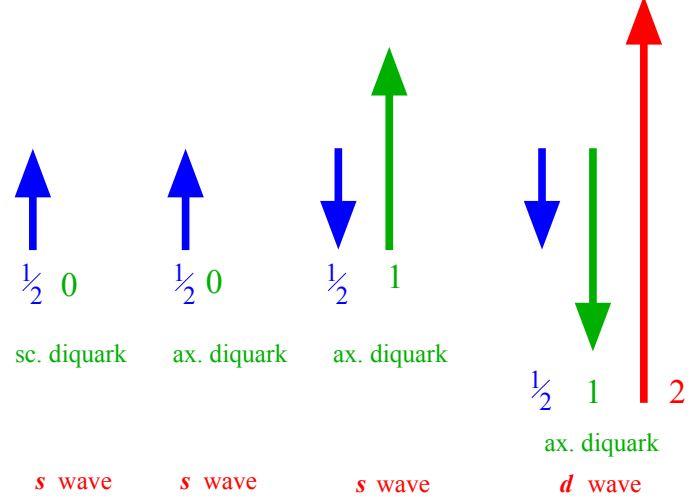

FIG. 27: Angular momentum coupling of the nucleon's three valence quarks.

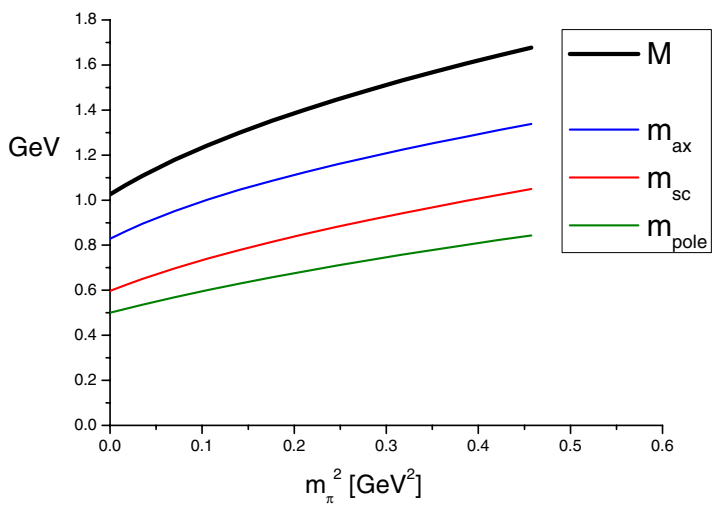

FIG. 28: The mass attributed to the nucleon's quark core, the diquark masses and the quark 'pole' mass as function of the pion mass squared. 


\section{Nucleon mass}

Solving the quark-diquark BSEs (for details of the numerical method see ref. [82]) one obtains a mass which can be attributed to the nucleon's quark core. This mass is above the physical value $M=0.94 \mathrm{GeV}$. Depending on the parameterization of the quark propagator and the calculated diquark masses the chiral limit value ranges between $M \sim 1.07 \mathrm{GeV}$ $\ldots 1.25 \mathrm{GeV}$. In fig. 28 this mass is displayed as a function of the pion mass squared together with diquark masses and the quark 'pole' mass. From fig. 29 it is evident that this overestimation of the nucleon mass is a result for all values of the current quark mass (or pion mass, respectively). The comparison to the results from Chiral Perturbation Theory and to lattice data [83] clearly shows that we missed some attractive contribution in the nucleon. A possible explanation which attributes this to missing pion effects can be found in refs. [84, 85].

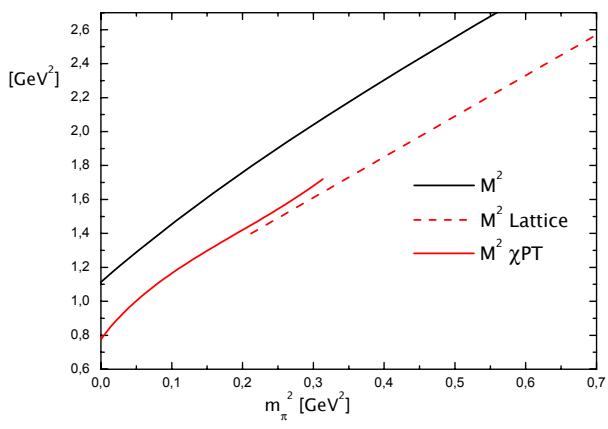

FIG. 29: The mass attributed to the nucleon's quark core compared to results from Chiral Perturbation Theory and lattice data [83] as function of the pion mass squared.

\section{Electromagnetic Current}

\section{Current Contributions}

To describe the coupling of the photon to the nucleon's quark core one has to construct the electromagnetic current operator. The corresponding five-point function has to fulfill a Ward-Takahashi identity which in turn guarantees that current conservation holds $[79,86]$. This requires to include at least the following diagrams (see fig. 30):

- photon-quark coupling,

- photon-diquark coupling,

- coupling to exchange quark,

- seagull terms: coupling to diquark amplitudes.

\begin{tabular}{c||c|c} 
& calc. & exp. \\
\hline \hline$r_{E}^{p}$ & 0.73 & 0.836 \\
$r_{E}^{n}$ & 0.29 & 0.336 \\
$r_{M}^{p}$ & 0.64 & 0.843 \\
$r_{M}^{n}$ & 0.60 & 0.840
\end{tabular}

TABLE II: The nucleons' electromagnetic radii (in fm) as calculated in the covariant Faddeev approach in comparison to experimental values.

\begin{tabular}{c||c|c|c} 
& calc. & exp. & without pions [95] \\
\hline \hline & & & \\
$\mu_{p}$ & 3.62 & 2.793 & $3.5 \pm 0.2$ \\
$\mu_{n}$ & -2.34 & -1.913 & $-2.6 \pm 0.2$ \\
$\kappa_{s}$ & 0.28 & -0.120 & -0.11 \\
$\kappa_{v}$ & 4.96 & 3.706 & $5.1 \pm 0.4$
\end{tabular}

TABLE III: The nucleons' magnetic moments (in n.m.) as calculated in the covariant Faddeev approach in comparison to experimental values.

Unknown constants like the anomalous magnetic moment of the axialvector diquark and the strength of the scalaraxialvector transitions will be calculated by taking into account the diquark substructure such that gauge invariance for the nucleon current is respected [87]. Due to the non-trivial momentum dependence of the quark propagator the quarkphoton vertex possesses a quite non-trivial structure [88-90]. This then also implies a rich structure of the diquark-photon vertex, for the construction of the latter and its use within a covariant Faddeev approach see e.g. refs. [91-94].

\section{Results on Electromagnetic Form Factors}

Equipped with the explicit form of the current operator the electromagnetic form factors can be calculated. For the corresponding results the reader is refered to [76], here the focus will be on derived quantities like the electric and magnetic radii as well as the magnetic moments.

From table II one sees that the nucleons' radii are underestimated, hereby the magnetic radii more than the electric ones. One can test the hypothesis whether this shortcoming is due to the missing of the pion cloud. First of all, in the isoscalar combination of the radii pion effects are of the second order and therefore small. A quantitative description of the nucleon's quark core should give values close to the experimental ones, and this is what we find. Second, at large pion mass the pion cloud effects should become less important, and our results should agree with lattice data. As can be seen from fig. 31 the agreement for the isovector part of the $F_{2}$ and $F_{1}$ radius is certainly not perfect but our results are not too far off the lattice data.

The absolute value of the magnetic moments is also too large. In addition to look at the isoscalar and isovector values one can compare directly to an estimate without pions [95], see table III, which is quite favourable for the interpretation of having mostly missed the pion cloud. Plotting the anomalous 

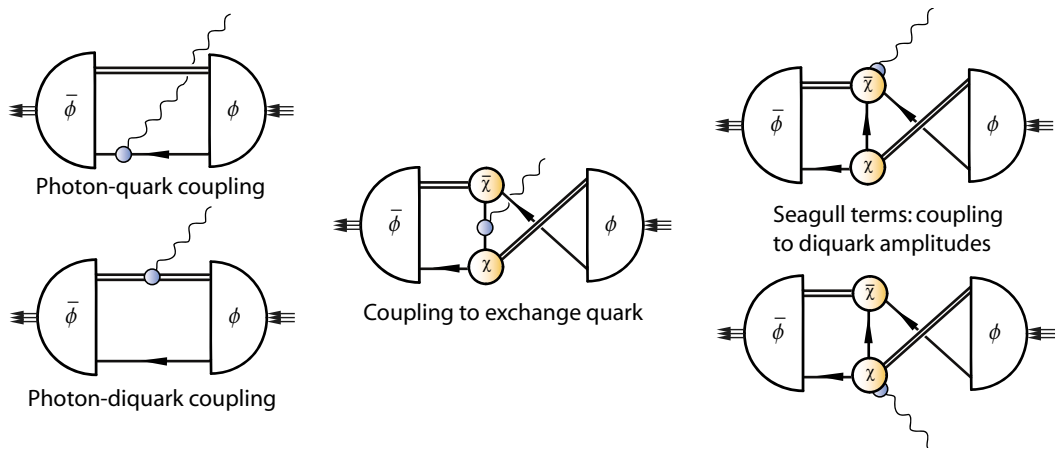

FIG. 30: Feyman diagrams which are taken into account in the construction of the nucleon's electromagnetic current operator.
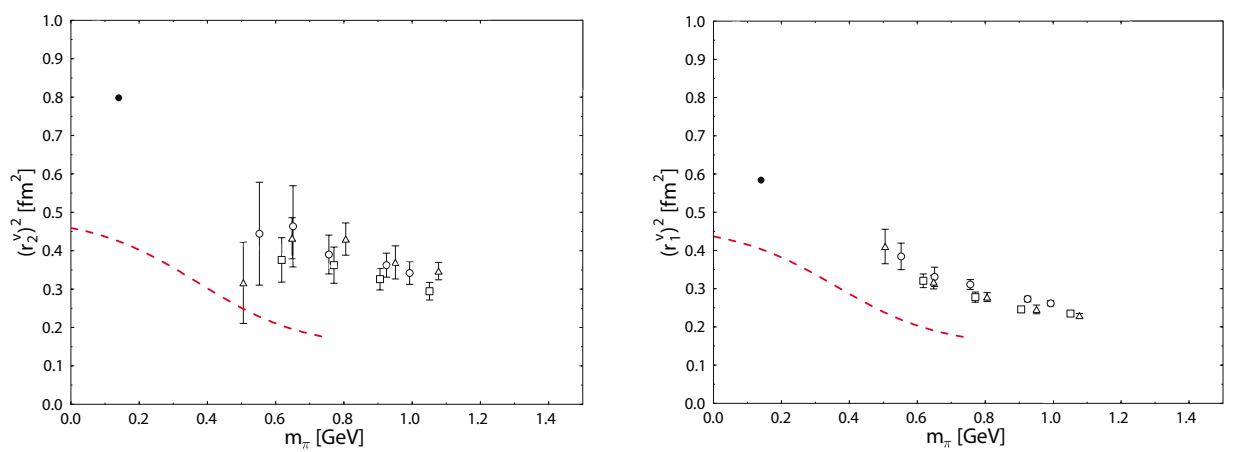

FIG. 31: The results for the $F_{2}$ isovector radius (left panel) and the $F_{1}$ isovector radius (right panel) as a function of the pion mass in comparison to the experimental value and corresponding lattice data [83].

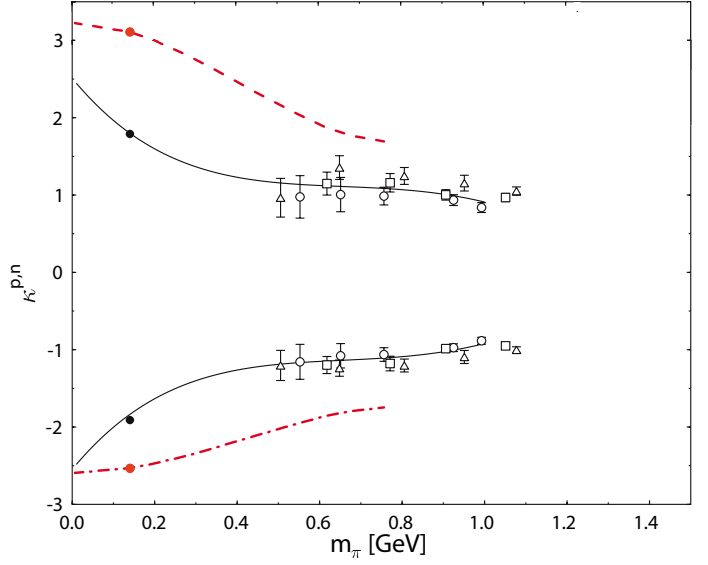

FIG. 32: The results for the anomalous magnetic moments (dashed lines) as a function of the pion mass in comparison to the experimental value and corresponding results from lattice data and their chiral extrapolations (fully drawn lines) [83].

magnetic moments against the pion mass and comparing to corresponding results from lattice data and their chiral extrapolation [83] provides further evidence for this interpretation.

\section{Summary of Part III}

The nucleons' mass, electromagnetic form factors, charge radii and magnetic moments have been calculated in a Poincaré covariant Faddeev approach

- employing dressed quarks and "diquarks", obtained from the quark DSE and diquark BSE (The corresponding propagators agree with lattice data where available.)

- as well as using dressed quark- and diquark-photon vertex functions.

- The results describe the nucleons' quark core without pion contributions and are obtained

- without model parameters, the only input scale is $\Lambda_{\mathrm{QCD}}\left(\Lambda_{\mathrm{MOM}}\right.$ into DSEs, $\Lambda_{\mathrm{L}}$ or $r_{0}$ in lattice calculations).

\section{OUTLOOK}

In these lectures I have attempted to demonstrate the progress made within functional approaches to strong continuum QCD and its application to hadronic physics. Of course, further improvements are possible and highly desirable at every step of these investigations. 
Within Landau gauge a consistent qualitative picture for all QCD propagators and vertex functions has emerged. First results within Coulomb gauge, see also the lectures by Dan Zwanziger, allow for a first more general interpretation of the results. The importance of the Gribov horizon in both gauges can possibly give a hint about the nature of confining field configurations.

Besides the fundamental issues of confinement and dynamical generation of masses QCD Green functions can serve as a first-principles input into a description of hadronic properties. The ideal calculations of this kind have the minimal parameter set of QCD and make real predictions. The results obtained so far allow for the optimistic view that a coherent description of hadronic states and processes based on the dynamics of confined quarks and Yang-Mills fields can be realized in the near future. Hereby different methods based on the QCD Green functions will yield complementary information. Lattice calculations provide evidence that truncations of the infinite hierarchy of Dyson-Schwinger and/or Renormalization Group equations have been done reasonably. On the other hand, results based on the full set of these equations as well as on truncated equations help to understand the lattice data.

To explain confinement is one of the truly fundamental challenges to contemporary physics. Understanding confinement will allow us to link the microscopic degrees of freedom of QCD, the quarks and gluons, to the measurable strong interactions in hadronic and nuclear physics. As long as the confinement phenomenon stays mysterious the standard model of particle physics lacks an important part, something essential in the fundamental laws of nature is then still unknown to us. Although Infrared $Q C D$ has posed us extremely challenging problems it is worth pursuing its research.

\section{Acknowledgments}

I cordially thank Attilio Cucchieri, Tereza Mendes and Silvio P. Sorella for inviting me to this extraordinarily interesting conference Infrared QCD in Rio and for their hospitality.

The work reported was done in collaboration with a number of colleagues and students. I am deeply thankful to all of them. I am especially grateful to Lorenz von Smekal and Christian Fischer for sharing their insights over many years with me and for the uncounted number of valuable discussions with them. I am greatly indebted to Will Detmold, Felipe Llanes-Estrada, Andreas Krassnigg, Axel Maas, Pieter Maris, Craig Roberts, Sebastian Schmidt and Pete Watson for their contributions to common research which deepened my understanding of Infared QCD and hadron physics.

Furthermore, I wish to thank Jeff Greensite, Štefan Olejník and Dan Zwanziger for many enlightening discussions about confinement, and Peter Tandy for helpful discussions about hadron physics.

This work is supported in part by the Deutsche Forschungsgemeinschaft under Grant No. A1279/5-1 and the Austrian Science Fund FWF under Grant No. W1203 (Doctoral Program "Hadrons in vacuum, nuclei and stars").
[1] R. Alkofer and L. von Smekal, Phys. Rept. 353, 281 (2001) [arXiv:hep-ph/0007355].

[2] C. S. Fischer, J. Phys. G: Nucl. Part. Phys. 32, R253 (2006) [arXiv:hep-ph/0605173].

[3] P. Maris and C. D. Roberts, Int. J. Mod. Phys. E 12, 297 (2003) [arXiv:nucl-th/0301049].

[4] R. Alkofer and J. Greensite, arXiv:hep-ph/0610365.

[5] D. J. Gross and A. Neveu, Phys. Rev. D 10, 3235 (1974).

[6] S. N. Gupta, Proc. Roy. Soc. A63, 681 (1950); K. Bleuler, Helv. Phys. Acta 23, 567 (1950).

[7] L. D. Faddeev and V. N. Popov, Phys. Lett. B 25, 29 (1967).

[8] C. Becchi, A. Rouet, and R. Stora, Annals Phys. 98, 287 (1976); see also: I. V. Tyutin, Lebedev preprint FIAN No. 39 (1975).

[9] T. Kugo and I. Ojima, Prog. Theor. Phys. Suppl. 66, 1 (1979).

[10] N. Nakanishi and I. Ojima, World Sci. Lect. Notes Phys. 27, 1 (1990).

[11] V. N. Gribov, Nucl. Phys. B 139, 1 (1978).

[12] see also: D. Zwanziger, Nucl. Phys. B 378, 525 (1992).

[13] P. van Baal, arXiv:hep-th/9711070.

[14] A. G. Williams, Nucl. Phys. Proc. Suppl. 109A, 141 (2002) [arXiv:hep-lat/0202010].

[15] A. Cucchieri, Nucl. Phys. B508, 353 (1997) [arXiv: heplat/9705005].

[16] F. D. Bonnet et al., Phys. Rev. D 64, 034501 (2001) [arXiv:heplat/0101013].

[17] C. Alexandrou, P. De Forcrand, and E. Follana, Phys. Rev. D 65, 117502 (2002) [arXiv:hep-lat/0203006].

[18] P. J. Silva and O. Oliveira, Nucl. Phys. B 690, 177 (2004)
[arXiv:hep-lat/0403026].

[19] S. Furui and H. Nakajima, Phys. Rev. D 70, 094504 (2004).

[20] P. O. Bowman et al., and A. G. Williams, Phys. Rev. D 70, 034509 (2004) [arXiv: hep-lat/0402032].

[21] A. Sternbecket al., Phys. Rev. D 72, 014507 (2005) [arXiv: hep-lat/0506007].

[22] D. Zwanziger, Phys. Rev. D 69, 016002 (2004) [arXiv:hep$\mathrm{ph} / 0303028]$.

[23] D. Zwanziger, Nucl. Phys. B 364, 127 (1991); Nucl. Phys. B 399, 477 (1993); Nucl. Phys. B 412, 657 (1994).

[24] H. Hata and I. Niigata, Nucl. Phys. B 389, 133 (1993) [arXiv: hep-ph/9207260].

[25] T. Mendes, A. Cucchieri, and A. Mihara, arXiv:heplat/0611002.

[26] T. Kugo, arXiv:hep-th/9511033.

[27] R. Oehme and W. Zimmermann, Phys. Rev. D 21, 471 (1980).

[28] R. Haag, Local Quantum Physics, Springer, 1996.

[29] P. Watson and R. Alkofer, Phys. Rev. Lett. 86, 5239 (2001) [arXiv:hep-ph/0102332].

[30] C. Lerche and L. von Smekal, Phys. Rev. D 65, 125006 (2002) [arXiv:hep-ph/0202194].

[31] D. Zwanziger, Phys. Rev. D 65, 094039 (2002) [arXiv:hepth/0109224].

[32] J. C. Taylor, Nucl. Phys. B 33, 436 (1971).

[33] A. Cucchieri, T. Mendes, and A. Mihara, JHEP 12, 012 (2004) [arXiv:hep-lat/0408034].

[34] W. Schleifenbaum et al., Phys. Rev. D 72, 014017 (2005) [arXiv:hep-ph/0411052]. 
[35] A. Sternbeck et al., PoS LAT2005, 333 (2005) [arXiv:heplat/0509090].

[36] R. Alkofer, C. S. Fischer, and F. J. Llanes-Estrada, Phys. Lett. B 611, 279 (2005) [arXiv:hep-th/0412330].

[37] C. S. Fischer and J. M. Pawlowski, Phys. Rev. D 75, 025012 (2007) [arXiv:hep-th/0609009].

[38] L. von Smekal, R. Alkofer, and A. Hauck, Phys. Rev. Lett. 79, 3591 (1997) [arXiv:hep-ph/9705242]; L. von Smekal, A. Hauck, and R. Alkofer, Annals Phys. 267, 1 (1998) [arXiv:hepph/9707327]; A. Hauck, L. von Smekal, and R. Alkofer, Comput. Phys. Commun. 112, 166 (1998) [arXiv:hep-ph/9804376].

[39] C. S. Fischer and R. Alkofer, Phys. Lett. B 536, 177 (2002) [arXiv:hep-ph/0202202]; C. S. Fischer, R. Alkofer, and H. Reinhardt, Phys. Rev. D 65, 0940082002 [arXiv:hepph/0202195]; R. Alkofer, C. S. Fischer, and L. von Smekal, Acta Phys. Slov. 52, 191 (2002) [arXiv:hep-ph/0205125].

[40] C. S. Fischer and R. Alkofer, Phys. Rev. D 67, 094020 (2003) [arXiv:hep-ph/0301094].

[41] J. M. Pawlowski et al., Phys. Rev. Lett. 93, 152002 (2004) [arXiv:hep-th/0312324].

[42] C. S. Fischer and H. Gies, JHEP 0410, 048 (2004) [arXiv:hep$\mathrm{ph} / 0408089]$

[43] O. Oliveira and P. J. Silva, these proceedings [arXiv:heplat/0609036].

[44] M. Müller-Preussker, these proceedings; A. Sternbeck, PhD Thesis, Humboldt Univ. Berlin, 2006 [arXiv:hep-lat/0609016].

[45] A. Maas, A. Cucchieri, and T. Mendes, these proceedings [arXiv:hep-lat/0610006].

[46] C. S. Fischer, B. Gruter, and R. Alkofer, Annals Phys. 321, 1918 (2006) [arXiv:hep-ph/0506053].

[47] C. S. Fischer et al., arXiv:hep-ph/0701050.

[48] J.B. Zhang et al., Phys. Rev. D 70, 034505 (2004) [arXiv:heplat/0301018].

[49] P. O. Bowman, U. M. Heller, and A. G. Williams, Phys. Rev. D 66, 014505 (2002) [arXiv:hep-lat/0203001].

[50] C. S. Fischer and R. Alkofer, AIP Conf. Proc. 756, 275 (2005) [arXiv:hep-ph/0411347].

[51] R. Alkofer et al., Phys. Rev. D 70, 014014 (2004) [arXiv:hepph/0309077]; Nucl. Phys. Proc. Suppl. 141, 122 (2005).

[52] D. V. Shirkov and I. L. Solovtsov, Phys. Rev. Lett. 79, 1209 (1997).

[53] A. Maas, J. Wambach, and R. Alkofer, Eur. Phys. J. C42, 93 (2005) [arXiv:hep-ph/0504019]; A. Maas et al., Eur. Phys. J. C37, 335 (2004) [arXiv:hep-ph/0408074];

[54] A. Cucchieri, T. Mendes, and A. R. Taurines, Phys. Rev. D 67, 091502 (2003) [arXiv:hep-lat/0302022].

[55] R. Alkofer, C. S. Fischer, and F. J. Llanes-Estrada, arXiv:hep$\mathrm{ph} / 0607293$.

[56] R. Alkofer et al., Phys. Rev. Lett. 96, 022001 (2006) [arXiv: hep-ph/0510028].

[57] K. G. Wilson, Phys. Rev. D 10, 2445 (1974).

[58] D. Zwanziger, Phys. Rev. Lett. 90, 102001 (2003).

[59] J. Greensite, S. Olejnik, and D. Zwanziger, Phys. Rev. D 69, 074506 (2004).

[60] A. Nakamura and T. Saito, Prog. Theor. Phys. 115, 189 (2006) [arXiv:hep-lat/0512042]

[61] A. P. Szczepaniak, Phys. Rev. D 69, 074031 (2004) [arXiv:hep$\mathrm{ph} / 0306030]$

[62] A. P. Szczepaniak and E. S. Swanson, AIP Conf. Proc. 549, 330 (2002).

[63] A. P. Szczepaniak and E. S. Swanson, Phys. Rev. D 65, 025012 (2002) [arXiv:hep-ph/0107078].
[64] D. Zwanziger, Phys. Rev. D 70, 094034 (2004) [arXiv:hep$\mathrm{ph} / 0312254]$

[65] C. Feuchter and H. Reinhardt, Phys. Rev. D 70, 105021 (2004) [arXiv:hep-th/0408236].

[66] J. R. Finger and J. E. Mandula, Nucl. Phys. B 199, 168 (1982).

[67] J. Govaerts et al., Nucl. Phys. B 237, 59 (1984).

[68] S. L. Adler and A. C. Davis, Nucl. Phys. B 244, 469 (1984).

[69] R. Alkofer and P. A. Amundsen, Nucl. Phys. B 306, 305 (1988).

[70] F. J. Llanes-Estrada et al., Phys. Rev. C 70, 035202 (2004) [arXiv:hep-ph/0402253].

[71] A. Cucchieri and D. Zwanziger, Phys. Rev. D 65, 014001 (2002) [arXiv:hep-lat/0008026].

[72] J. Greensite, Prog. Part. Nucl. Phys. 51, 1 (2003) [arXiv:heplat/0301023]

[73] J. Greensite and S. Olejnik, Phys. Rev. D 67, 094503 (2003) [arXiv:hep-lat/0302018].

[74] J. Greensite, S. Olejnik, and D. Zwanziger, arXiv:heplat/0410028.

[75] K. Langfeld, R. Alkofer, and P. A. Amundsen, Z. Phys. C 42, 159 (1989).

[76] G. Eichmann, Diploma Thesis, Graz University 2006, Advisors: R. Alkofer and A. Krassnigg [http://physik.uni-graz.at/ itp/DD/eichmann/diplomarbeit.pdf].

[77] R. Alkofer and M. Oettel, talk given at the Schladming Winter School 2005 [arXiv:nucl-th/0507003].

[78] M. Oettel et al., Phys. Rev. C 58, 2459 (1998) [arXiv:nuclth/9805054]; G. Hellstern et al., Nucl. Phys. A 627, 679 (1997) [arXiv:hep-ph/9705267]

[79] M. Oettel, M. Pichowsky, and L. von Smekal, Eur. Phys. J. A 8, 251 (2000) [arXiv:nucl-th/9909082].

[80] M. Oettel, PhD thesis, Tübingen University 2000, Advisor: R. Alkofer [arXiv:nucl-th/0012067].

[81] R. Alkofer, G. Eichmann, and A. Krassnigg, in preparation.

[82] M. Oettel, L. von Smekal, and R. Alkofer, Comput. Phys. Commun. 144, 63 (2002) [arXiv:hep-ph/0109285].

[83] M. Gockeler et al. [QCDSF Collaboration], Phys. Rev. D 71, 034508 (2005) [arXiv:hep-lat/0303019].

[84] M. B. Hecht et al., Phys. Rev. C 65, 055204 (2002) [arXiv:nuclth/0201084].

[85] M. Oettel and A. W. Thomas, Phys. Rev. C 66, 065207 (2002) [arXiv:nucl-th/0203073].

[86] A. N. Kvinikhidze and B. Blankleider, Phys. Rev. C 60, 044003 (1999) [nucl-th/9901001].

[87] M. Oettel, R. Alkofer, and L. von Smekal, Eur. Phys. J. A 8, 553 (2000) [arXiv:nucl-th/0006082].

[88] J. S. Ball and T. Chiu, Phys. Rev. D 22, 2542 (1980).

[89] D. C. Curtis and M. R. Pennington, Phys. Rev. D 42, 4165 (1990).

[90] P. Maris and P. C. Tandy, Phys. Rev. C 61, 045202 (2000) [nuclth/9910033].

[91] C. Weiss et al., Phys. Lett. B 312, 6 (1993) [arXiv:hep$\mathrm{ph} / 9305215]$.

[92] S. Ahlig et al., Phys. Rev. D 64, 014004 (2001) [arXiv:hep$\mathrm{ph} / 0012282]$.

[93] M. Oettel and R. Alkofer, Eur. Phys. J. A 16, 95 (2003) [arXiv:hep-ph/0204178].

[94] R. Alkofer et al., Few Body Syst. 37, 1 (2005) [arXiv:nuclth/0412046]; A. Höll et al., Nucl. Phys. A 755, 298 (2005) [arXiv:nucl-th/0501033].

[95] T. R. Hemmert and W. Weise, Eur. Phys. J. A 15, 487 (2002) [arXiv:hep-lat/0204005]. 